Article

\title{
Static Isolated Horizons: $S U(2)$ Invariant Phase Space, Quantization, and Black Hole Entropy
}

\section{Alejandro Perez ${ }^{\star}$ and Daniele Pranzetti}

Centre de Physique Théorique, Université de Provence, Campus de Luminy, 13288, Marseille, France; E-Mail: pranzetti@cpt.univ-mrs.fr

* Author to whom correspondence should be addressed; E-Mail: perez@cpt.univ-mrs.fr.

Received: 22 February 2011; in revised form: 16 March 2011 / Accepted: 16 March 2011 /

Published: 25 March 2011

\begin{abstract}
We study the classical field theoretical formulation of static generic isolated horizons in a manifestly $S U(2)$ invariant formulation. We show that the usual classical description requires revision in the non-static case due to the breaking of diffeomorphism invariance at the horizon leading to the non-conservation of the usual pre-symplectic structure. We argue how this difficulty could be avoided by a simple enlargement of the field content at the horizon that restores diffeomorphism invariance. Restricting our attention to static isolated horizons we study the effective theories describing the boundary degrees of freedom. A quantization of the horizon degrees of freedom is proposed. By defining a statistical mechanical ensemble where only the area $a_{H}$ of the horizon is fixed macroscopically — states with fluctuations away from spherical symmetry are allowed-we show that it is possible to obtain agreement with the Hawkings area law $\left(S=a_{H} /\left(4 \ell_{p}^{2}\right)\right)$ without fixing the Immirzi parameter to any particular value: consistency with the area law only imposes a relationship between the Immirzi parameter and the level of the Chern-Simons theory involved in the effective description of the horizon degrees of freedom.
\end{abstract}

Keywords: quantum gravity; black hole entropy; isolated horizon 


\section{Introduction}

One of the most striking predictions of General Relativity $(\mathrm{GR})$ is the creation of Black Holes $(\mathrm{BH})$ as the final stage of gravitational collapse. A prediction that is by now supported by an important accumulation of observational evidence [1-3]. However, despite the extremely simple general relativistic description of the spacetime geometry of the black hole $(\mathrm{BH})$ external region-at least in the idealized scenario given by the Kerr-Newman solutions describing the situation once the dynamical phase of collapse has settled down - the power of general relativity reduces drastically when it comes to the understanding of the internal dynamics, as singularities of the spacetime geometry are unavoidably developed. Simple arguments imply that a complete consistent description of the gravitational collapse would necessarily require dealing with quantum fluctuations of the gravitational interaction described by some quantum theory of gravity.

However, not only the interior BH physics - unaccessible to external observers-is calling for a theory of quantum gravity (QG). As shown by the celebrated works of Bekenstein and Hawking ([4,5]), there are strong theoretical arguments indicating that idealized BHs in their stationary phase behave as thermodynamical systems, with their own analogue of the zeroth, first, second, and third law of thermodynamics, respectively. More precisely one has that: $(0)$ The surface gravity $\kappa_{H}$ on the event horizon of stationary BH is constant. (1) Under external perturbation the initially stationary state of a black hole can change but the final stationary state will be described by another Kerr-Newman solution whose parameters (mass $M$, electric charge $Q$ and angular momentum $J$ ) readjust according to

$$
\delta M=\frac{\kappa_{H}}{8 \pi G} \delta a_{H}+\Phi_{H} \delta Q+\Omega_{H} \delta J
$$

where $a_{H}$ is the horizon area, $\Phi_{H}$ is the electrostatic potential at the horizon, and $\Omega_{H}$ the angular velocity of the horizon. (2) The BH horizon area can only increase

$$
\delta a_{H} \geq 0
$$

(3) No finite physical process can allow a BH to become extremal $\left(\kappa_{H}=0\right)$.

This analogy with thermodynamics became strict with the discovery of the Hawking effect which implies that stationary BHs radiate as black bodies with a temperature $T=\kappa_{H} /(2 \pi)$ and hence, through the first law, have an entropy $S=a_{H} /\left(4 \ell_{p}^{2}\right)$. Understanding the physical nature of the microscopic degrees of freedom leading to such entropy requires a quantum description of the gravitational field. In this paper we study the problem from the perspective of loop quantum gravity [6-9].

However, the very notion of black hole-as the region causally disconnected from future null infinity-becomes elusive in the context of quantum gravity due to the simple fact that black hole radiation in the semiclassical regime imply that in the full quantum theory the global structure of space-time (expected to make sense away from the strong field region) might completely change. In fact, recent models in two dimensions support the view that this is the case [10]. For that reason, the problem of black hole entropy in quantum gravity requires the use of a local or quasi-local notion of horizon in equilibrium.

In recent years such a local definition of $\mathrm{BH}$ has been introduced ([11]) through the concept of Isolated Horizons (IH). Isolated horizons are regarded as a sector of the phase-space of GR containing 
a horizon in "equilibrium" with the external matter and gravitational degrees of freedom. This local definition has been first used for the black-hole entropy calculation (for spherically symmetric IH) in [12] in the context of loop quantum gravity. In this seminal work the authors show, after introduction of a suitable gauge fixing, how the degrees of freedom that are relevant for the entropy calculation can be encoded in a boundary $U(1)$ Chern-Simons theory. Based on this work, state counting of horizon states leads to agreement with the Bekenstein-Hawking formula upon appropriate tuning of the Immirzi parameter [13-20] (for a complete description of the beautiful counting techniques used in these calculations see [21]).

A more simple, natural and effective description of the boundary degrees of freedom for spherically symmetric IH can be obtained in terms of an $S U(2)$ Chern-Simons theory [22,23]. This latter treatment clarifies how the $U(1)$ gauge reduction previously used leads to the over counting of allowed horizon states [22]. As a consequence the entropy calculation changes: agreement with the area law requires the tuning of the Immirzi parameter to a different value, and the universal logarithmic corrections change from $\Delta S_{U(1)}=-1 / 2 \log a_{H}$ to $\Delta S_{S U(2)}=-3 / 2 \log a_{H}$ now in agreement with other approaches [24-31]. However, there remains the somewhat unnatural spherical symmetry requirement at the classical level that one would like to eliminate.

In this work we extend the $S U(2)$ invariant treatment to a wider class of IH containing distortion. More precisely we consider generic isolated horizons [32,33] of the static type and show how they too admit an effective description in terms of $S U(2)$ Chern-Simons theories similar in spirit to what was found in the spherically symmetric case. Our approach is fundamentally different from the one of [34] where - thanks to the additional assumption of axisymmetry (not necessary in the present treatment) - the system is mapped to a model equivalent to the Type I case if the multipole moments describing the amount of distortion are fixed classically. Recently the treatment of [34] has been generalized to generic isolated horizons in [35] in a way that allows to remove all symmetry assumptions, where the main idea remains to describe the boundary degrees of freedom in terms of a canonical connection (called area-connection in the second reference). In the present treatment, no symmetry assumption is necessary either (Type I, Type II, and Type III horizons are all treated on equal footing), only staticity is a necessary condition for the dynamical system to be well defined (see Section 4). Our approach is different from previous work dealing with distorted IH [34,35] in two main respects: first the treatment is $S U(2)$ gauge invariant avoiding in this way the difficulties found upon quantization in the gauge fixed $U(1)$ formulation, and second, distortion is not erased by the choice of a mapping to a canonical Type I connection. In particular, the degrees of freedom related to distortion are encoded in observables of our system and can be quantized. In this new treatment we can find the old Type I theory in the sense that when we define the statistical mechanical ensemble by fixing the macroscopic area $a_{H}$ and imposing spherical symmetry, we get an entropy consistent with the one in [23]. Moreover, we can go beyond this observation computing the entropy for an ensemble where only the area is fixed macroscopically while distortion is allowed to fluctuate (see also [35]).

We will show that a one-parameter ambiguity arises when one describes the classical boundary theory in terms of $S U(2)$ connections. This ambiguity is analogous to the appearance of the Immirzi parameter in the description of the phase space of general relativity in terms of $S U(2)$ connection variables. More precisely, the horizon degrees of freedom will be described by a pair of $S U(2)$ Chern-Simons theories 
with the same level $k$ which is otherwise arbitrary. Hence, the classical ambiguity referred to above is encoded in the value of the level $k \in \mathbb{N}$ of the Chern-Simons theories. We show that one can recover the Bekenstein-Hawking entropy without the need of fixing the Immirzi parameter to a particular value. Instead, (the semiclassical) Hawking's area law 'dictates' the relationship between the Immirzi parameter in the bulk theory (LQG) and the analog in the boundary theory (the Chern-Simons level), in the sense that the Immirzi parameter can now take different discrete values according to the choice of the level $k \in \mathbb{N}$.

The paper is organized as follows: In Section 2 we restate the definition of isolated horizons as put in [23] and based on [32,33], and include a new classification of isolated horizons according to the reality of the Weyl tensor component $\Psi_{2}$ (this classification is already implicit in [32,33]). In Section 3 we introduce the basic equations that follow from the definition of isolated horizons. In Section 4 we construct the pre-symplectic structure of static isolated horizons and prove its conservation. We also show that the usual treatment used so far in the literature on isolated horizons cannot be directly applied in the case of non-static horizons and requires a modification. We propose a possible way to deal with this problem; however, a full analysis of this issue is outside the scope of this paper. In Section 5 we quantize the system and show that the subset of spherically symmetric states in the Hilbert space are in one-to-one correspondence with the admissible states in the formulation of reference [23]. Thus, if spherical symmetry is imposed as a condition defining the ensemble of states in the statistical mechanical treatment, the entropy comes out proportional to $a_{H}$ as expected. In Section 6 we compute the entropy starting from an ensemble where only the area is fixed macroscopically and distortion is allowed, we show that compatibility with the area law can be obtained and, if an appropriate and natural paradigm shift is undertaken, there is no need to fix the Immirzi parameter to a given specific value (Subsection 6.2). Concluding remarks are presented in Section 7.

\section{Definition of Isolated Horizons}

There are some parts of this section that literally follow reference [23].

The standard definition of a $\mathrm{BH}$ as a spacetime region from which no information can reach idealized observers at (future null) infinity is a global definition. This notion of $\mathrm{BH}$ requires a complete knowledge of a spacetime geometry and is therefore not suitable for describing local physics. On physical grounds a quasilocal definition is necessary for quantum considerations. That this should be the case is clear from the fact that physical black holes are expected to radiate due to the Hawking effect and hence the usual mathematical definition might not even make sense. The first quasilocal definitions in loop quantum gravity were introduced in $[11,32,33]$ with the name of isolated horizons (IH). Here we present this definition according to $[32,33,36,37]$. A full discussion of the geometrical meaning of the following conditions can be found in $[23,32,33]$.

Definition: The internal boundary $\Delta$ of a history $\left(\mathscr{M}, g_{a b}\right)$ will be called an isolated horizon provided the following conditions hold:

(i) Manifold conditions: $\Delta$ is topologically $S^{2} \times R$, foliated by a (preferred) family of 2-spheres equipped with an equivalence class $\left[\ell^{a}\right]$ of transversal future pointing null vector fields whose flow preserves the foliation, where $\ell^{a}$ is equivalent to $\ell^{\prime a}$ if $\ell^{a}=c \ell^{\prime a}$ for some positive real number $c$. 
(ii) Dynamical conditions: All field equations hold at $\Delta$.

(iii) Matter conditions: On $\Delta$ the stress-energy tensor $T_{a b}$ of matter is such that $-T_{b}^{a} \ell^{b}$ is causal and future directed.

(iv) Conditions on the metric $g$ determined by e, and on its levi-Civita derivative operator $\nabla$ : (iv.a) The expansion of $\ell^{a}$ within $\Delta$ is zero. This, together with the energy condition (iii) and the Raychaudhuri equation at $\Delta$, ensures that $\ell^{a}$ is additionally shear-free. This in turn implies that the Levi-Civita derivative operator $\nabla$ naturally determines a derivative operator $D_{a}$ intrinsic to $\Delta$ via $X^{a} D_{a} Y^{b}:=X^{a} \nabla_{a} Y^{b}, X^{a}, Y^{a}$ tangent to $\Delta$. We then impose (iv.b) $\left[\mathscr{L}_{\ell}, D\right]=0$.

(v) Restriction to 'good cuts.' One can show furthermore that $D_{a} \ell^{b}=\omega_{a} \ell^{b}$ for some $\omega_{a}$ intrinsic to $\Delta$. A 2-sphere cross-section $H$ of $\Delta$ is called a 'good cut' if the pull-back of $\omega_{a}$ to $H$ is divergence free with respect to the pull-back of $g_{a b}$ to $H$. As shown in [32,33], every horizon satisfying (i)-(iv) above possesses at least one foliation into 'good cuts'; this foliation is furthermore generically unique. We require that the fixed foliation coincide with a foliation into 'good cuts.'

\subsection{Isolated Horizon Classification According to Their Symmetry Groups}

Next, let us examine symmetry groups of isolated horizons. A symmetry of $\left(\Delta, q, D,\left[\ell^{a}\right]\right)$ is a diffeomorphism on $\Delta$ which preserves the horizon geometry $(q, D)$ and at most rescales elements of $\left[\ell^{a}\right]$ by a positive constant. It is clear that diffeomorphisms generated by $\ell^{a}$ are symmetries. So, the symmetry group $G_{\Delta}$ is at least 1-dimensional. In fact, there are only three possibilities for $G_{\Delta}$ :

(a) Type I: the isolated horizon geometry is spherical; in this case, $G_{\Delta}$ is four dimensional $(S O(3)$ rotations plus rescaling-translations along $\ell$, which, in a coordinate system where $\ell^{a}=(\partial / \partial v)^{a}$, corresponds to the affine map $v \rightarrow c v+b$ with $c, b \in \mathbb{R}$ constants);

(b) Type II: the isolated horizon geometry is axi-symmetric; in this case, $G_{\Delta}$ is two dimensional (rotations round symmetry axis plus rescaling-translations along $\ell$ );

(c) Type III: the diffeomorphisms generated by $\ell^{a}$ are the only symmetries; $G_{\Delta}$ is one dimensional.

Note that these symmetries refer only to the horizon geometry. The full space-time metric need not admit any isometries even in a neighborhood of the horizon.

\subsection{Isolated Horizons Classification According to the Reality of $\Psi_{2}$}

(a) Static: In the Newman-Penrose formalism (in the null tetrads adapted to the IH geometry introduced in the following section) static isolated horizons are characterized by the condition

$$
\operatorname{Im}\left(\Psi_{2}\right)=0
$$

on the Weyl tensor component $\Psi_{2}=C_{a b c d} \ell^{a} m^{b} \bar{m}^{c} n^{d}$. One can then show that for this class of isolated horizons (see next section) the pull-back to $H$ (the preferred family of sections) of $v\lrcorner K_{i} \Sigma^{i}$ vanishes for all $v \in T(H)$. This corresponds to having the horizon locally "at rest". In the axisymmetric case, according to the definition of multiple moments of Type II horizons constructed in [34], static isolated horizons are non-rotating isolated horizons, i.e., those for which all angular momentum multiple moments vanish. Static black holes (e.g., those in the Reissner-Nordtrom family) have static isolated horizons. There are Type I, II and III static isolated horizons. 
(b) Non-Static: In the Newman-Penrose formalism (in the null tetrads adapted to the IH geometry introduced in the following section) non-static isolated horizons are characterized by the condition

$$
\operatorname{Im}\left(\Psi_{2}\right) \neq 0
$$

The pull-back to $H$ (the preferred family of sections) of $v\lrcorner K_{i} \Sigma^{i}$ does not vanish for all $v \in T(H)$. The horizon is locally "in motion". The Kerr black hole is an example of this type.

In this paper we will construct the conserved pre-symplectic structure of static isolated horizons (no symmetry assumptions on the horizon are made). We will also show that the usual pre-symplectic structure is not conserved in the presence of a non-static black hole, which implies that a complete treatment of non-static isolated horizons (including rotating isolated horizons) remains open. In this direction, we propose some general ideas leading to a conserved symplectic structure for non-static isolated horizons and the restoration of diffeomorphism invariance. However, due to the very different nature of such approach, the quantization of such proposals is left for future studies.

\section{Some Key Equations}

In this section we use the definition of isolated horizons provided in the previous section to prove some of the equations we will need in the sequel. General relativity in the first order formalism is described in terms a tetrad of four 1-forms $e^{I}\left(I=0,3\right.$ internal indices) and a Lorentz connection $\omega^{I J}=-\omega^{J I}$. The metric can be recovered by

$$
g_{a b}=e_{a}^{I} e_{b}^{J} \eta_{I J}
$$

where $\eta_{I J}=\operatorname{diag}(-1,1,1,1)$. In the time gauge, where the tetrad $e^{I}$ is such that $e^{0}$ is a time-like vector field normal to $M$, the three 1 -forms $K^{i}=\omega^{0 i}$ play a special role in the parametrization of the phase space. In particular the so-called Ashtekar connection is

$$
A_{a}^{+i}=\Gamma_{a}^{i}+i K_{a}^{i}
$$

where $\Gamma^{i}$ is the spin connection satisfying Cartan's first equation $d_{\Gamma} e^{i}=0$. We also introduce

$$
\Sigma^{I J} \equiv e^{I} \wedge e^{J} \text { and } \Sigma^{+i} \equiv \epsilon^{i}{ }_{j k} \Sigma^{j k}+2 i \Sigma^{0 i}
$$

and $F^{i}(A)$ the curvature of the connection $A^{i}$. At $H$, we will also often work in the gauge where $e^{1}$ is normal to $H$ and $e^{2}$ and $e^{3}$ are tangent to $H$. This choice is only made for convenience, as the equations are all gauge covariant, their validity in one frame implies their validity in all frames.

Statement 1: In the gauge where the tetrad is chosen so that $\ell^{a}=2^{-1 / 2}\left(e_{0}^{a}+e_{1}^{a}\right)$ (which can be completed to a null tetrad $n^{a}=2^{-1 / 2}\left(e_{0}^{a}-e_{1}^{a}\right)$, and $\left.m^{a}=2^{-1 / 2}\left(e_{2}^{a}+i e_{3}^{a}\right)\right)$, the shear-free and vanishing expansion (condition (iv.a) in the definition of $\mathrm{IH}$ ) imply

$$
\stackrel{\omega^{21}}{\Leftarrow}=\stackrel{\omega}{\omega}^{20} \text { and } \stackrel{\omega^{31}}{\Leftarrow}=\omega^{30}
$$

where the double arrow means "pull-back to the horizon". The proof of this statement can be found in [23]. 
Statement 2: We start from the identity (that can be derived from Cartan's second structure equations)

$$
F_{a b}^{i}\left(A^{+}\right)=-\frac{1}{4} R_{a b}^{c d} \Sigma_{c d}^{+i}
$$

where $R_{a b c d}$ is the Riemann tensor and $\Sigma^{+i}=\epsilon_{j k}^{i} e^{j} \wedge e^{k}+i 2 e^{0} \wedge e^{i}$. A simple algebraic calculation using the null tetrad formalism (see for instance [40] page 43) with the null tetrad of Statement 1, and the definitions $\Psi_{2}=C_{a b c d} \ell^{a} m^{b} \bar{m}^{c} n^{d}$ and $\Phi_{11}=R_{a b}\left(\ell^{a} n^{b}+m^{a} \bar{m}^{b}\right) / 4$, where $R_{a b}$ is the Ricci tensor and $C_{a b c d}$ the Weyl tensor, yields

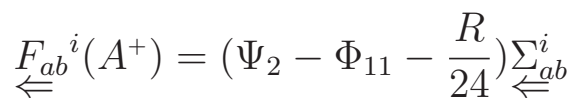

where, on $M, \Sigma^{i}=\operatorname{Re}\left[\Sigma^{+i}\right]=\epsilon_{j k}^{i} e^{j} \wedge e^{k}$. For simplicity, here we will assume that no matter is present at the horizon, $\Phi_{11}=R=0$, hence

$$
F_{a b}^{F^{i}}\left(A^{+}\right)=\Psi_{2} \underset{\sum_{a b}^{i}}{\Leftarrow}
$$

An important point here is that the previous expression is valid for any two sphere $S^{2}$ (not necessarily a horizon) embedded in spacetime in an adapted null tetrad where $\ell^{a}$ and $n^{a}$ are normal to $S^{2}$.

Here we will concentrate, for simplicity, in the pure gravity case. In this special case, and due to the vanishing of both the expansion and shear of the generators congruence $\ell^{a}$, the Weyl component $\Psi_{2}$ at the Horizon is simply related to the Gauss scalar curvature $R^{(2)}$ of the two spheres

Statement 3: The statement 1 has an immediate consequence for static isolated horizons: the reality of $\Psi_{2}$ implies, for the component $i=1$ in the frame of the statement 1 , that $d K^{1}=0$. The good-cut condition $(v)$ in the definition then implies that

$$
\stackrel{K}{K}^{1}=0
$$

Statement 4: For static isolated horizons we have that

$$
\stackrel{K^{j}}{\Leftarrow} \stackrel{K^{k}}{\Leftarrow} \epsilon_{i j k}=c \stackrel{\sum^{i}}{\Leftarrow}
$$

for $c: H \rightarrow \mathbb{R}$ an extrinsic curvature scalar.

Proof: In order to simplify the notation all free indices associated to forms that appear in this proof are pulled back to $H$ (this allows us to drop the double arrows from equations). In the frame of statement 1 , where $e^{1}$ is normal to $H$, the only non-trivial component of the equation we want to prove is the $i=1$ component, namely:

$$
K^{A} \wedge K^{B} \epsilon_{A B}=c \Sigma^{1}
$$

where $A, B=2,3$ and $\epsilon^{A B}=\epsilon^{1 A B}$. Now, in that gauge, we have that $K^{A}=c_{B}^{A} e^{B}$ for some matrix of coefficients $c_{B}^{A}$. Notice that the left hand side of the previous equation equals $\operatorname{det}(c) e^{A} \wedge e^{B} \epsilon_{A B}$. We only need to prove that $\operatorname{det}(c)$ is time independent, i.e., that $\ell(\operatorname{det} c)=0$. We need to use the isolated horizon boundary condition

$$
\left[\mathscr{L}_{\ell}, D_{b}\right] v^{a}=0 \quad v^{a} \in T(\Delta)
$$

where $D_{a}$ is the derivative operator determined on the horizon by the Levi-Civita derivative operator $\nabla_{a}$. One important property of the commutator of two derivative operators is that it also satisfy the Leibnitz 
rule (it is itself a new derivative operator). Therefore, using the fact that the null vector $n^{a}$ is normalized so that $\ell \cdot n=-1$ we get

$$
0=\left[\mathscr{L}_{\ell}, D_{b}\right] \ell^{a} n_{a}=n_{a}\left[\mathscr{L}_{\ell}, D_{b}\right] \ell^{a}+\ell^{a}\left[\mathscr{L}_{\ell}, D_{b}\right] n_{a} \quad \Rightarrow \quad \ell^{a}\left[\mathscr{L}_{\ell}, D_{b}\right] n_{a}=0
$$

where we have also used that $\ell^{a} \in T(\Delta)$. Now, if one introduces a coordinate $v$ on $\Delta$ such that $\ell^{a} \partial_{a} v=1$ and $v=0$ on some leaf of the foliation, then it follows - from the fact that $\ell$ is a symmetry of the horizon geometry $(q, D)$, and the fact that the horizon geometry uniquely determines the foliation into 'good cuts'-that $v$ will be constant on all the leaves of the foliation. As $n$ must be normal to the leaves one has $n=-d v$, whence $d n=0$. From this it follows that $\left.\left.\mathscr{L}_{\ell} n=\ell\right\lrcorner d n+d(\ell\lrcorner n\right)=0$ and, therefore, evaluating Equation (15) on the right hand side explicitly, we get

$$
\begin{aligned}
0 & =\ell^{a}\left[\mathscr{L}_{\ell}, D_{b}\right] n_{a}=\ell^{a} \mathscr{L}_{\ell}\left(D_{b} n_{a}\right)=-\frac{1}{\sqrt{2}} \ell^{a} \mathscr{L}_{\ell}\left(D_{b}\left[e_{a}^{1}+e_{a}^{0}\right]\right) \\
& =\frac{1}{\sqrt{2}} \ell^{a} \mathscr{L}_{\ell}\left(\omega_{b \mu}^{1} e_{a}^{\mu}+\omega_{b \mu}^{0} e_{a}^{\mu}\right)=-\frac{1}{\sqrt{2}} \ell^{a} \mathscr{L}_{\ell}\left(\omega_{b}^{10}\left[e_{a}^{0}+e_{a}^{1}\right]\right)+\frac{1}{\sqrt{2}} \ell^{a} \mathscr{L}_{\ell}\left(\omega_{b A}^{1} e_{a}^{A}+\omega_{b A}^{0} e_{a}^{A}\right) \\
& =\ell^{a} \mathscr{L}_{\ell}\left(\omega_{b}^{10}\right) n_{a}
\end{aligned}
$$

where in the second line we have used the fact that $D_{b} e_{a}^{\nu}=-\omega_{b \mu}^{\nu} e_{a}^{\mu}$ plus the fact that as $\mathscr{L}_{\ell} q_{a b}=0$ the Lie derivative $\mathscr{L}_{\ell} e^{A}=\alpha \epsilon^{A B} e_{B}$ for some $\alpha$ (moreover, one can even fix $\alpha=0$ if one wanted to by means of internal gauge transformations). Then it follows that

$$
\mathscr{L}_{\ell} K^{1}=0
$$

a condition that is also valid for the so called weakly isolated horizons [32,33]. A similar argument as the one given in Equation (15)_but now replacing $\ell^{a}$ by $e_{B}^{a} \in T(\Delta)$ for $B=2,3$ - leads to

$$
\begin{aligned}
0 & =e_{B}^{a}\left[\mathscr{L}_{\ell}, D_{b}\right] n_{a}=e_{B}^{a} \mathscr{L}_{\ell}\left(D_{b} n_{a}\right)=-\frac{1}{\sqrt{2}} e_{B}^{a} \mathscr{L}_{\ell}\left(D_{b}\left[e_{a}^{1}+e_{a}^{0}\right]\right) \\
& =\frac{1}{\sqrt{2}} e_{B}^{a} \mathscr{L}_{\ell}\left(\omega_{b \mu}^{1} e_{a}^{\mu}+\omega_{b \mu}^{0} e_{a}^{\mu}\right)=-\frac{1}{\sqrt{2}} e_{B}^{a} \mathscr{L}_{\ell}\left(\omega_{b}^{10}\left[e_{a}^{0}+e_{a}^{1}\right]\right)+\frac{1}{\sqrt{2}} e_{B}^{a} \mathscr{L}_{\ell}\left(\omega_{b A}^{1} e_{a}^{A}+\omega_{b A}^{0} e_{a}^{A}\right) \\
& =\sqrt{2} e_{B}^{a} \mathscr{L}_{\ell}\left(\omega_{b A}^{0} e_{a}^{A}\right)=\sqrt{2}\left[\mathscr{L}_{\ell}\left(\omega_{b}^{0 B}\right)+\alpha \epsilon^{B A} \omega_{b}^{0 A}\right]
\end{aligned}
$$

where, in addition to previously used identities, we have made use of statement 1, Equation (7). The previous equations imply that the left hand side of Equation (13) is Lie dragged along the vector field $\ell$, and since $\Sigma^{i}$ is also Lie dragged (in this gauge), all this implies that $\mathscr{L}_{\ell}(\operatorname{det}(c))=\ell(\operatorname{det}(c))=0$.

Remark 1: In the GHP formalism [39], a null tetrad formalism compatible with the choice of tetrad of statement 1 and hence the IH system, the scalar curvature of the two-spheres normal to $\ell^{a}$ and $n^{a}$ is given by

$$
R^{(2)}=K+\bar{K}
$$

where $K=\sigma \sigma^{\prime}-\rho \rho^{\prime}-\Psi_{2}+R+\Phi_{11}$, while $\sigma, \rho, \sigma^{\prime}$ and $\rho^{\prime}$ denote spin shear and expansion spin coefficients associated with $\ell^{a}$ and $n^{a}$ respectively. The shear-free and expansion-free conditions in the definition of IHs translate into $\rho=0=\sigma$ in the GHP formalism, namely

$$
R^{(2)}=-2 \Psi_{2}
$$


Similarly, the curvature scalar $c$ in (12) can be expressed in terms of spin coefficients as

$$
c=\frac{1}{2}\left(\rho^{\prime} \bar{\rho}^{\prime}-\sigma^{\prime} \bar{\sigma}^{\prime}\right)
$$

which is invariant under null tetrad transformations fixing $\ell^{a}$ and $n^{a}$.

Remark 2: The quantity $d \equiv 2 \Psi_{2}+c$ will play a central role in what follows. From the discussion above we observe that it can be rewritten as

$$
d=c-R^{(2)}
$$

i.e., it is given by the difference between Gauss scalar curvature of the horizon-encoding the local intrinsic geometry of the cross-sections-and the extrinsic curvature invariant $c$. This quantity will be often referred to as the distortion of static isolated horizon in the rest of this paper. In the entropy calculation we will considered an statistical mechanical ensemble where only the horizon area is fixed macroscopically, thus, states with all possible values of the above local quantity (allowed by the quantum theory) are counted.

\section{The Conserved Symplectic Structure}

In this section we prove the conservation of the symplectic structure of gravity in the presence of an isolated horizon that is not necessarily spherically symmetric. In this sense, our proof generalizes the one presented in [23].

\subsection{The Action Principle}

The action principle of general relativity in self dual variables containing an inner boundary satisfying the IH boundary condition (for asymptotically flat spacetimes) takes the form

$$
S\left[e, A^{+}\right]=-\frac{i}{\kappa} \int_{\mathscr{M}} \Sigma_{i}^{+}(e) \wedge F^{i}\left(A^{+}\right)+\frac{i}{\kappa} \int_{\tau_{\infty}} \Sigma_{i}^{+}(e) \wedge A^{+i}
$$

where a boundary contribution at a suitable time cylinder $\tau_{\infty}$ at infinity is required for the differentiability of the action. No boundary term is necessary if one allows variations that fix an isolated horizon geometry up to diffeomorphisms and Lorentz transformations [23].

First variation of the action yields

$$
\delta S\left[e, A^{+}\right]=\frac{-i}{\kappa} \int_{\mathscr{M}} \delta \Sigma_{i}^{+}(e) \wedge F^{i}\left(A^{+}\right)-d_{A^{+}} \Sigma_{i}^{+} \wedge \delta A^{+i}+d\left(\Sigma_{i}^{+} \wedge \delta A^{+i}\right)+\frac{i}{\kappa} \int_{\tau_{\infty}} \delta\left(\Sigma_{i}^{+}(e) \wedge A^{+i}\right)
$$

from which the self dual version of Einstein's equations follow

$$
\begin{aligned}
& \epsilon_{i j k} e^{j} \wedge F^{i}\left(A^{+}\right)+i e^{0} \wedge F_{k}\left(A^{+}\right)=0 \\
& e_{i} \wedge F^{i}\left(A^{+}\right)=0 \\
& d_{A^{+}} \Sigma_{i}^{+}=0
\end{aligned}
$$

as the boundary terms in the variation of the action cancel. 


\subsection{The Conserved Symplectic Structure in Terms of Vector Variables}

In this work we study general relativity on a spacetime manifold with an internal boundary satisfying the boundary condition corresponding to static isolated horizons, and asymptotic flatness at infinity. The phase space of such system is denoted $\Gamma$ and is defined by an infinite dimensional manifold where points $p \in \Gamma$ are given by solutions to Einstein's equations satisfying the static IH boundary conditions. Explicitly a point $p \in \Gamma$ can be parametrized by a pair $p=\left(\Sigma^{+}, A^{+}\right)$satisfying the field equations (23) and the requirements of Definition 2. In particular fields at the boundary satisfy Einstein's equations and the constraints given in Section 3. Let $\mathrm{T}_{\mathrm{p}}(\Gamma)$ denote the space of variations $\delta=\left(\delta \Sigma^{+}, \delta A_{+}\right)$at $p$ (in symbols $\left.\delta \in \mathrm{T}_{\mathrm{p}}(\Gamma)\right)$.

So far we have defined the covariant phase space as an infinite dimensional manifold. For it to become a phase space it is necessary to provide it with a presymplectic structure. As the field equations, the presymplectic structure can be obtained from the first variation of the action (22). In particular a symplectic potential density for gravity can be directly read off from the total differential term in (22) $[41,42]$. In terms of the Ashtekar connection and the densitized tetrad, the symplectic potential density is

$$
\theta(\delta)=\frac{-i}{\kappa} \Sigma_{i}^{+} \wedge \delta A^{+i} \quad \forall \delta \in T_{p} \Gamma
$$

where $\kappa=16 \pi G$ and the symplectic current takes the form

$$
J\left(\delta_{1}, \delta_{2}\right)=-\frac{2 i}{\kappa} \delta_{[1} \Sigma_{i}^{+} \wedge \delta_{2]} A^{+i} \quad \forall \delta_{1}, \delta_{2} \in T_{p} \Gamma
$$

Einstein's equations imply $d J=0$. From Stokes theorem applied to the four dimensional (shaded) region in Figure 1 bounded by $M_{1}$ in the past, $M_{2}$ in the future, a timelike cylinder at spacial infinity on the right, and the isolated horizon $\Delta$ on the left we obtain

$$
\delta \mu \equiv-i \int_{M_{1}} \Sigma_{i} \wedge \delta\left(\Gamma^{i}+i K^{i}\right)+i \int_{M_{2}} \Sigma_{i} \wedge \delta\left(\Gamma^{i}+i K^{i}\right)+i \int_{\Delta} \Sigma_{i}^{+} \wedge \delta A^{+i}
$$

for some functional $\mu$. As we will show now, for static isolated horizons, the previous equation implies that symplectic form

$$
\kappa \Omega_{M}\left(\delta_{1}, \delta_{2}\right)=\int_{M} \delta_{[1} \Sigma^{i} \wedge \delta_{2]} K_{i}
$$

is conserved in the sense that $\Omega_{M_{2}}\left(\delta_{1}, \delta_{2}\right)=\Omega_{M_{1}}\left(\delta_{1}, \delta_{2}\right)$. In order to prove the above statement it is sufficient to show that

$$
\delta \tilde{\mu}=\int_{M_{1}} \Sigma_{i} \wedge \delta K^{i}-\int_{M_{2}} \Sigma_{i} \wedge \delta K^{i}
$$

for some functional $\tilde{\mu}$.

The isolated horizon boundary condition imply that the only variations of the fields allowed on the horizon $\Delta$ are infinitesimal $S L(2, \mathbb{C})$ gauge transformations and diffeomorphisms, namely:

$$
\begin{aligned}
& \delta e=\delta_{\alpha} e+\delta_{v} e \\
& \delta A^{+}=\delta_{\alpha} A^{+}+\delta_{v} A^{+} \\
& \delta K=\delta_{\alpha} K+\delta_{v} K
\end{aligned}
$$


where $\alpha: M \rightarrow s l(2, \mathbb{C})$ and $v$ is a vector field tangent to $H$. Under such transformations we have:

$$
\begin{gathered}
\delta_{\alpha} e^{i}=[\alpha, e]^{i} \quad \delta_{\alpha} \Sigma^{+i}=\left[\alpha, \Sigma^{+}\right]^{i} \quad \delta_{\alpha} A^{+i}=-d_{A^{+}} \alpha^{i} \\
\left.\left.\delta_{v} e^{i}=v\right\lrcorner d e^{i}+d(v\lrcorner e^{i}\right) \\
\left.\left.\delta_{v} A^{+i}=v\right\lrcorner F^{i}\left(A^{+}\right)+d_{A^{+}}(v\lrcorner A^{+i}\right) \\
\left.\left.\delta_{v} \Sigma^{+i}=d_{A^{+}}(v\lrcorner \Sigma^{+}\right)^{i}-[v\lrcorner A^{+}, \Sigma^{+}\right]^{i}
\end{gathered}
$$

where $(v\lrcorner \omega)_{b_{1} \cdots b_{p-1}} \equiv v^{a} \omega_{a b_{1} \cdots b_{p-1}}$ for any $p$-form $\omega_{b_{1} \cdots b_{p}}$, and in the last line we used the Gauss law. Let us also recall the some useful relations:

$$
A \wedge v\lrcorner B=-v\lrcorner A \wedge B
$$

for any 2-form $A$ and 1-form $B$ on a 2-manifold or any 2-form $A$ and 2-form $B$ on a 3-manifold, while

$$
A \wedge v\lrcorner B=v\lrcorner A \wedge B
$$

for any 1-form $A$ and 2-form $B$ on a 2-manifold or any 1-form $A$ and 3-form $B$ on a 3-manifold. We used the notation $\Gamma^{i}=-\frac{1}{2} \epsilon^{i}{ }_{j k} \omega^{j k}$ when working with the torsion free connection compatible with the triad $e^{i}$. In this notation the torsion free condition (Cartan's first structure equation) takes the form

$$
d e^{i}=-\epsilon^{i}{ }_{j k} \Gamma^{j} \wedge e^{k}
$$

which implies

$$
F^{i}(\Gamma)=d \Gamma^{i}+\frac{1}{2} \epsilon^{i}{ }_{j k} \Gamma^{j} \wedge \Gamma^{k}
$$

A very important property of the spin connection [6] compatible with $e^{i}$ is that

$$
\int_{M} \Sigma_{i} \wedge \delta \Gamma^{i}=\int_{H}-e_{i} \wedge \delta e^{i}
$$

This identity allows one to rewrite (26) as

$$
\delta \mu-i \mathscr{D}(\delta)=\int_{M_{1}} \Sigma_{i} \wedge \delta K^{i}-\int_{M_{2}} \Sigma_{i} \wedge \delta K^{i}
$$

where

$$
\mathscr{D}(\delta)=\int_{H_{1}-H_{2}} e_{i} \wedge \delta e^{i}-\int_{\Delta} \Sigma_{i}^{+} \wedge \delta A^{+i}
$$

More specifically, we can evaluate the previous equation on the allowed variations on the boundary. For gauge transformations we get

$$
\begin{aligned}
i \mathscr{D}\left(\delta_{\alpha}\right) & =i \int_{H_{1}-H_{2}} e_{i} \wedge[\alpha, e]^{i}+i \int_{\Delta} d\left(\Sigma_{i}^{+} \alpha^{i}\right)-d \Sigma^{+}{ }_{i} \alpha^{i}+\Sigma^{+}{ }_{i} \wedge \epsilon_{j k}^{i} A^{+j} \alpha^{k} \\
& =i \int_{H_{1}-H_{2}} e_{i} \wedge \epsilon^{i}{ }_{j k} \alpha^{j} e^{k}+i \int_{\Delta} d\left(\Sigma^{+}{ }_{i} \alpha^{i}\right)-d \Sigma^{+}{ }_{i} \alpha^{i}-\epsilon_{i j k} A^{+j} \wedge \Sigma^{+k} \alpha^{i} \\
& =-i \int_{H_{1}-H_{2}} \Sigma_{i} \alpha^{i}+i \int_{H_{1}-H_{2}} \Sigma_{i} \alpha^{i}-i \int_{\Delta} d_{A^{+}} \Sigma^{+}{ }_{i} \alpha^{i}=0
\end{aligned}
$$


where in the last line we have used the Gauss law. Therefore, for gauge transformations on the horizon, we have proven the result (28). Let us now analyze the diffeomorphisms. Analogously to the gauge transformations case, we have

$$
\begin{aligned}
i \mathscr{D}\left(\delta_{v}\right) & \left.\left.\left.\left.=i \int_{H_{1}-H_{2}} e_{i} \wedge(v\lrcorner d e^{i}+d(v\lrcorner e^{i}\right)\right)-i \int_{\Delta} \Sigma^{+}{ }_{i} \wedge(v\lrcorner F^{i}\left(A^{+}\right)+d_{A^{+}}(v\lrcorner A^{+i}\right)\right) \\
& \left.\left.\left.\left.=i \int_{H_{1}-H_{2}} 2 v\right\lrcorner e_{i} d e^{i}-d\left(e_{i} v\right\lrcorner e^{i}\right)-i \int_{\Delta} d\left(\Sigma^{+}{ }_{i} v\right\lrcorner A^{+i}\right)-d_{A^{+}} \Sigma^{+}{ }_{i} v\right\lrcorner A^{+i} \\
& \left.\left.=i \int_{H_{1}-H_{2}} \Sigma_{i} v\right\lrcorner \Gamma^{i}-i \int_{H_{1}-H_{2}} \Sigma_{i} v\right\lrcorner A^{+i} \\
& \left.\left.=\int_{H_{1}-H_{2}} \Sigma_{i} v\right\lrcorner K^{i}=-\int_{H_{1}-H_{2}} v\right\lrcorner \Sigma_{i} \wedge K^{i}
\end{aligned}
$$

where in the first line we have used the vector constraint, in the second one the Gauss law and in the third one the Cartan equation. Therefore, in the case of diffeomorphisms for the variations on the horizon, the result (28) holds as long as

$$
v\lrcorner \Sigma_{i} \wedge K^{i}=0
$$

If the previous relation is satisfied, i.e., if the isolated horizon is static. From (28) it follows that the conserved symplectic form for gravity can be written as:

$$
\kappa \Omega\left(\delta_{1}, \delta_{2}\right)=\int_{M} \delta_{[1} \Sigma^{i} \wedge \delta_{2]} K_{i}
$$

where $M$ is a Chauchy surface representing space and $\delta_{1}, \delta_{2} \in T_{p} \Gamma$, i.e., they are vectors in the tangent space to the phase-space $\Gamma$ at the point $p$. The symplectic form above is manifestly real and has no boundary contribution.

Figure 1. The characteristic data for a (vacuum) spherically symmetric isolated horizon corresponds to Reissner-Nordstrom data on $\Delta$, and free radiation data on the transversal null surface with suitable fall-off conditions. For each mass, charge, and radiation data in the transverse null surface there is a unique solution of Einstein-Maxwell equations locally in a portion of the past domain of dependence of the null surfaces. This defines the phase space of Type I isolated horizons in Einstein-Maxwell theory. The picture shows two Cauchy surfaces $M_{1}$ and $M_{2}$ "meeting" at space-like infinity $i_{0}$. A portion of $\mathscr{I}^{+}$and $\mathscr{I}^{-}$are shown; however, no reference to future time-like infinity $i^{+}$is made as the isolated horizon need not to coincide with the black hole event horizon.

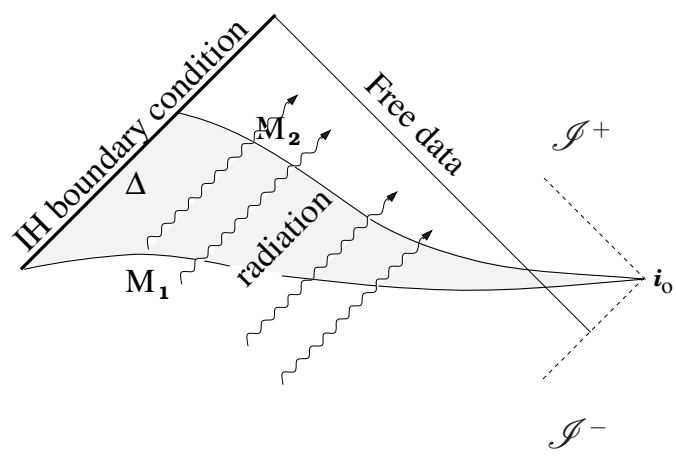




\subsection{Gauge Symmetries}

The gauge symmetry content of isolated horizon system is characterized by the degenerate directions of the presymplectic structure. In fact we now show how tangent vectors $\delta_{\alpha}, \delta_{v} \in T_{p} \Gamma$ of the form

$$
\begin{aligned}
& \delta_{\alpha} \Sigma=[\alpha, \Sigma], \quad \delta_{\alpha} K=[\alpha, K] ; \\
& \left.\left.\left.\left.\delta_{v} \Sigma=\mathscr{L}_{v} \Sigma=v\right\lrcorner d \Sigma+d(v\lrcorner \Sigma\right), \quad \delta_{v} K=\mathscr{L}_{v} K=v\right\lrcorner d K+d(v\lrcorner K\right)
\end{aligned}
$$

for $\alpha: M \rightarrow \mathfrak{s u}(2)$ and $v \in \operatorname{Vect}(M)$ tangent to the horizon, are degenerate directions of $\Omega_{M}$ if an only if the isolated horizon is static.

The proof given here follows exactly the one presented in [23]. However, our analysis here applies to generic static isolated horizons instead of simply Type I isolated horizons. We start with the $S U(2)$ transformations $\delta_{\alpha}$, and we get

$$
\left.\kappa \Omega_{M}\left(\delta_{\alpha}, \delta\right)=\int_{M}[\alpha, \Sigma]_{i} \wedge \delta K^{i}-\delta \Sigma_{i} \wedge[\alpha, K]^{i}=\int_{M} \delta\left(\epsilon_{i j k} \alpha^{j} \Sigma^{k} \wedge K^{i}\right)\right)=0
$$

where we used the Gauss constraint $\epsilon_{i j k} \Sigma^{k} \wedge K^{i}=0$. In order to treat the case of the infinitesimal diffeomorphisms tangent to the horizon $H$ it will be convenient to first write the form of the vector constraint $V_{a}$ in terms of $\Sigma-K$ variables [48]. We have

$$
\left.\left.v\lrcorner V=d K^{i} \wedge v\right\lrcorner \Sigma_{i}+v\right\lrcorner K^{i} d \Sigma_{i} \approx 0
$$

variations of the previous equation yields

$$
\begin{aligned}
v\lrcorner \delta V & \left.\left.\left.\left.=d(\delta K)^{i} \wedge v\right\lrcorner \Sigma_{i}+d K^{i} \wedge v\right\lrcorner \delta \Sigma_{i}+v\right\lrcorner \delta K^{i} d \Sigma_{i}+v\right\lrcorner K^{i} d(\delta \Sigma)_{i} \\
& \left.\left.\left.=v\lrcorner \Sigma_{i} \wedge d(\delta K)^{i}-\delta \Sigma_{i} \wedge v\right\lrcorner d K^{i}+v\right\lrcorner d \Sigma_{i} \wedge \delta K^{i}+d(\delta \Sigma)_{i} v\right\lrcorner K^{i}=0
\end{aligned}
$$

where in the second line we have put all the $K$ 's to the right, and modified the second and third terms using the identities (32)-(33). We are now ready to show that $\delta_{v}$ is a null direction of $\Omega_{M}$. Explicitly:

$$
\begin{aligned}
& \left.\left.\left.\left.\kappa \Omega_{M}\left(\delta_{v}, \delta\right)=\int_{M}(v\lrcorner d \Sigma+d(v\lrcorner \Sigma\right)\right)_{i} \wedge \delta K^{i}-\delta \Sigma_{i} \wedge(v\lrcorner d K+d(v\lrcorner K\right)\right)^{i} \\
& \left.\left.\left.\left.=\int_{M} v\right\lrcorner d \Sigma_{i} \wedge \delta K^{i}+d(v\lrcorner \Sigma\right)_{i} \wedge \delta K^{i}-\delta \Sigma^{i} \wedge v\right\lrcorner d K_{i}-\delta \Sigma_{i} \wedge d(v\lrcorner K\right)^{i} \\
& =\int_{M} \underbrace{\left.\left.\left.v\lrcorner d \Sigma_{i} \wedge \delta K^{i}+v\right\lrcorner \Sigma_{i} \wedge d(\delta K)^{i}-\delta \Sigma^{i} \wedge v\right\lrcorner d K_{i}+d(\delta \Sigma)^{i} \wedge v\right\lrcorner K_{i}}_{v\lrcorner \delta V=0} \\
& \left.\left.\left.+\int_{\partial M} v\right\lrcorner \Sigma_{i} \wedge \delta K^{i}-\delta \Sigma_{i} \wedge v\right\lrcorner K^{i}=\int_{\partial M} \delta(v\lrcorner \Sigma_{i} \wedge K^{i}\right)=0
\end{aligned}
$$

where in the last line we have used again the identity (32), the fact that $v$ is tangent to $H$, and the $\mathrm{IH}$ staticity condition (3) - from where (11) follows—implying that $v\lrcorner \Sigma_{i} \wedge K^{i}=0$ when pulled back to the horizon.

\subsection{The Conserved Symplectic Structure for Non-Static Isolated Horizons}

We have seen that the symplectic structure (42) is conserved if and only if the isolated horizon is static. The last equation clearly shows how this fails in the non-static case as (42) breaks diffeomorphisms 
invariance: tangent diffeomorphisms cease to be degenerate directions of the presymplectic structure and hence can no longer be interpreted as a gauge symmetry of the phase space. This is indeed an important point as it implies that the usual techniques for quantization and calculation of entropy are not applicable for non static isolated horizons which contain in particular the Kerr black hole. There are two ways out of this difficulty:

1. To declare that diffeomorphism are not gauge symmetries in the non-static case and modify the definition of the phase space allowing variations of fields at the horizon which are only pure $S U(2)$ gauge transformations (i.e., what would correspond to $v=0$ in (29)).

2. To restore diffeomorphism invariance at the horizon by the inclusion of new field degrees of freedom.

The first possibility appears as the simplest way out, and it is indeed a natural one often used in other contexts. For instance this is done in the construction of the phase space of asymptotically flat spacetimes where the boundary conditions reduce the gauge symmetries to global symmetries allowing in this way the definition of non-trivial charges such us linear and angular momentum at infinity. However, this approach is not viable in the present context where one intends to define a model capable of accounting for the degrees of freedom leading to black hole entropy. The reason is that diffeomorphism invariance is a central ingredient for the consistency of the quantum theory describing the degrees of freedom at the horizon and their relationship with the bulk loop quantum gravity degrees of freedom. The lack of diffeomorphism invariance in the phase space of the isolated horizon makes the usual program inapplicable.

Consequently, here we make a small step in the direction of the second possibility and propose a concrete extension of the phase space restring the conservation of the symplectic structure with a simple extension of (29). As it is well known and used in other contexts, diffeomorphism invariance can be restored by introducing additional degrees of freedom on the horizon. For instance, in the simpler context of $2+1$ gravity coupled to particles, one initially breaks diffeomorphism and gauge invariance at points. The degrees of freedom of the coupled particles are naturally defined by the requirement that the gauge symmetries are restored which necessitates the extension of the field content at the position of the particles. In our case, concretely, one should replace (42) by

$$
\kappa \Omega_{n s}\left(\delta_{1}, \delta_{2}\right)=\int_{M} \delta_{[1} \Sigma^{i} \wedge \delta_{2]} K_{i}+\int_{H} \delta_{[1} J \wedge \delta_{2]} \phi
$$

where $J$ is a 2-form field and $\phi$ a scalar field which under infinitesimal diffeomorphisms tangent to $H$ transform in the usual way, namely

$$
\left.\left.\delta_{v} J=d(v\lrcorner J\right) \quad \delta_{v} \phi=v\right\lrcorner d \phi
$$

We see immediately that

$$
\begin{aligned}
\kappa \Omega_{n s}\left(\delta_{v}, \delta\right) & \left.\left.\left.=\int_{\partial M} \delta(v\lrcorner \Sigma_{i} \wedge K^{i}\right)+d(v\lrcorner J\right) \wedge \delta \phi-\delta J \wedge v\right\lrcorner d \phi \\
& \left.\left.=\int_{\partial M} \delta(v\lrcorner \Sigma_{i} \wedge K^{i}-J \wedge v\right\lrcorner d \phi\right)
\end{aligned}
$$


which would vanish as desired if we imposed the restriction

$$
\left.v\lrcorner \Sigma_{i} \wedge K^{i}-J \wedge v\right\lrcorner d \phi=0
$$

on the new fields. The previous constraint is nothing else but the diffeomorphism constraint of the new extended system. In the axisymmetric case (e.g., the Kerr solution) $\phi=\varphi$ and the previous equation implies $J=\Sigma_{i} \wedge K_{\varphi}^{i}$ which is precisely the angular momentum density, indeed for Kerr [40], one has

$$
M a=\int_{H} J=\int_{H} \Sigma_{i} \wedge K_{\varphi}^{i}
$$

where $M$ is the mass and $a$ is the angular momentum per unit mass of the Kerr spacetime.

This trick allows us for restoring diffeomorphism invariance but the question remains: is the new symplectic structure (48) conserved for a non-static isolated horizon? The answer is affirmative as the following calculation shows. We can simply adapt the conservation proof given in Section 4.2 to this case. Indeed, if we start from Equation (37) which is valid for generic isolated horizons and we add on both sides the same term

$$
\int_{H_{1}-H_{2}} J \wedge \delta \phi
$$

we get

$$
\delta \mu-i \mathscr{D}_{n s}(\delta)=\int_{M_{1}} \Sigma_{i} \wedge \delta K^{i}+\int_{H_{1}} J \wedge \delta \phi-\int_{M_{2}} \Sigma_{i} \wedge \delta K^{i}-\int_{H_{2}} J \wedge \delta \phi
$$

where

$$
\mathscr{D}_{n s}(\delta)=\int_{H_{1}-H_{2}}\left(e_{i} \wedge \delta e^{i}+J \wedge \delta \phi\right)-\int_{\Delta} \Sigma^{+}{ }_{i} \wedge \delta A^{+i}
$$

As $\delta_{\alpha} J=0=\delta_{\alpha} \phi$, for gauge transformations we get

$$
i \mathscr{D}_{n s}\left(\delta_{\alpha}\right)=0
$$

Let us now analyze the diffeomorphisms;

$$
\left.\left.i \mathscr{D}_{n s}\left(\delta_{v}\right)=\int_{H_{1}-H_{2}}-v\right\lrcorner \Sigma_{i} \wedge K^{i}+J \wedge v\right\lrcorner d \phi=0
$$

according to (51). Therefore, the new symplectic structure (48) is conserved and diffeomorphism invariance at the boundary is restored with the addition of the new fields $(J, \phi)$. This extended system is now suitable to be quantized in consistency with the bulk theory. The understanding of the BH entropy calculation for rotating black holes could be based on this formulation. The quantization and interpretation of this new system is clearly a more difficult problem that we hope to study in more detail in the future.

\subsection{The Conserved Symplectic Structure in Terms of Real Connection Variables}

We now want to introduce the Ashtekar-Barbero variables

$$
A_{a}^{i}=\Gamma_{a}^{i}+\beta K_{a}^{i}
$$


where $\Gamma^{i}=-\frac{1}{2} \epsilon^{i j k} \omega_{j k}$ and $\beta$ is the Immirzi parameter. We can write the symplectic potential corresponding to (42) as

$$
\begin{aligned}
\kappa \Theta(\delta) & =\frac{1}{\beta} \int_{M} \Sigma_{i} \wedge \delta\left(\Gamma^{i}+\beta K^{i}\right)-\frac{1}{\beta} \int_{M} \Sigma_{i} \wedge \delta \Gamma^{i} \\
& =\frac{1}{\beta} \int_{M} \Sigma_{i} \wedge \delta\left(\Gamma^{i}+\beta K^{i}\right)+\frac{1}{\beta} \int_{H} e_{i} \wedge \delta e^{i}
\end{aligned}
$$

where in the last line we have used (36). In terms of the Ashtekar-Barbero connection the symplectic structure (42) takes the form:

$$
\kappa \Omega_{M}\left(\delta_{1}, \delta_{2}\right)=\frac{1}{\beta} \int_{M} \delta_{[1} \Sigma^{i} \wedge \delta_{2]} A_{i}-\frac{1}{\beta} \int_{H} \delta_{[1} e^{i} \wedge \delta_{2]} e_{i}
$$

where $H$ is the boundary of $M$.

Let us now comment on the nature of this result. We have shown that in the presence of a static isolated horizon the conserved pre-symplectic structure is the usual one when written in terms of vector-like (or Palatini) variables. When we write the pre-symplectic structure in terms of Ashtekar-Barbero connection variables in the bulk, the pre-symplectic structure acquires a boundary term at the horizon of the simple form $[23,43]$

$$
\kappa \Omega_{H}\left(\delta_{1}, \delta_{2}\right)=\frac{1}{\beta} \int_{H} \delta_{[1} e^{i} \wedge \delta_{2]} e_{i}
$$

A first observation is that, as shown in [23], this implies the kind of non-commutativity of flux variables that is compatible with the use of the holonomy-flux algebra as the starting point for quantization. This (completely continuum classical analysis) reinforces in this sense the importance of the kinematical quantization at the basis of the definition of loop quantum gravity [44,45]. A second observation is that the degrees of freedom at the horizon are encoded in the pull back of the triad fields $e^{i}$ on the horizon subjected to the obvious constraint

$$
\Sigma_{H}^{i}=\Sigma_{B u l k}^{i}
$$

which are three first class constraints—as it follows from (60)_for the six unconstrained phase space variables $e^{i}$. Therefore, one would expect that (as in the Type I case [22,23]) all of the boundary degrees of freedom are fixed by the bulk degrees of freedom. However, this turn out not to be that case if one works with triad fields as fundamental. The difficulty appears in the quantum theory where degenerate geometries are admitted. This can be visualized by concentrating on the special case $\Sigma_{H}^{i}=0$-that would correspond in the quantum theory to points where there is no bulk spin-network puncture. It is easy to see that the constraints $\Sigma_{H}^{i}=0$ does not kill all the local degrees of freedom in $e^{i}$ as there is a non-trivial moduli space of degenerate $e^{i}$ s that would naively lead to an infinite entropy. This can be made precise by studying in more detail the quantization of the $e^{i}$ fields on $H$. This more detailed analysis offers interesting possibilities beyond the scope of this work. Results are going to be reported elsewhere [46].

This difficulty is clearly related to the choice of continuum variables used for the parametrization of the boundary phase space. For instance, in the spherically symmetric case, the degrees of freedom are encoded instead in a connection $A^{i}$ and the analog of the constraints $\Sigma_{H}^{i}=0$ (where there are no bulk punctures) are $F^{i}(A)=0$. The dimensionality of both the unconstrained phase space and 
constraint surfaces are the same as in the treatment based on triads; however, the constraint $F^{i}(A)=0$ completely annihilates the local degrees of freedom at places where there are no punctures-as it implies that $A=g d g^{-1}$ (pure gauge) locally-rendering the entropy finite. This motivates the use of connection variables to describe (60). One of the results of this paper is to show that this can indeed be achieved for generic IH by the introduction of a pair of connection variables

$$
A_{\gamma}^{i}=\Gamma^{i}+\gamma e^{i} \text { and } A_{\sigma}^{i}=\Gamma^{i}+\sigma e^{i}
$$

in terms of which the boundary term of the conserved symplectic form (60) becomes

$$
\kappa \beta \Omega_{H}\left(\delta_{1}, \delta_{2}\right)=\frac{1}{\sigma^{2}-\gamma^{2}} \int_{H} \delta_{[1} A_{\gamma}^{i} \wedge \delta_{2]} A_{\gamma i}-\frac{1}{\sigma^{2}-\gamma^{2}} \int_{H} \delta_{[1} A_{\sigma}^{i} \wedge \delta_{2]} A_{\sigma i}
$$

The proof of this statement is presented in the following subsection. From the IH boundary conditions, through the relations (10) and (12), Cartan equations, and the definitions (62), the following relations for the new variables follow

$$
\begin{aligned}
& F^{i}\left(A_{\gamma}\right)=\Psi_{2} \Sigma^{i}+\frac{1}{2}\left(\gamma^{2}+c\right) \Sigma^{i} \\
& F^{i}\left(A_{\sigma}\right)=\Psi_{2} \Sigma^{i}+\frac{1}{2}\left(\sigma^{2}+c\right) \Sigma^{i}
\end{aligned}
$$

This means that there is a two-parameter family of equivalent classical descriptions of the system that in terms of triad variables is described by (60) (we will see in the sequel that the two parameter freedom reduces indeed to a single one when additional consistency requirements are taken into account). The appearance of these new parameters $\sigma$ and $\gamma$ is strictly related with the introduction of the $S U(2)$ connection variables (as was already observed in [23]). In this sense the situation is completely analogous to the one leading to the appearance of the Immirzi parameter when going from vector (Palatini) variables to Ashtekar-Barbero variables in the parametrization of the phase space of general relativity in the bulk.

In the quantum theory, at points where there are no punctures from the bulk, the two connections are subjected to the six first class constraints $F^{i}\left(A_{\gamma}\right)=0=F^{i}\left(A_{\sigma}\right)$ implying the absence of local degrees of freedom at these places. The new variables resolve in this way the difficulty that we encountered in the treatment in terms of the triads $e^{i}$. In addition, the connection fields $A_{\gamma}$ and $A_{\sigma}$ are described by Chern-Simons symplectic structures respectively, which will allow the use of some of the standard techniques, applicable to Type I isolated horizons, for the quantization of arbitrary static isolated horizons.

Remark: Using the well known relationship between Chern-Simons theory and $2+1$ gravity [50,51] it is possible to rewrite (63) in terms of $2+1$ gravity like variables: an $S U(2)$ connection and a 'triad' field. However, the coupling constraints (64) become more cumbersome in the prospect of quantization.

\subsection{Equivalence between the Triad and Connection Parametrizations of the Boundary Symplectic Structure}

In this section we present the proof of the validity of Equation (63). The key point—-from which (63) follows directly_-is to show that phase space one-form $\Theta_{0}(\delta)$ defined by

$$
\Theta_{0}(\delta) \equiv \int_{H} e_{i} \wedge \delta e^{i}-\frac{1}{\sigma^{2}-\gamma^{2}} \int_{H}\left(A_{\gamma i} \wedge \delta A_{\gamma}^{i}-A_{\sigma i} \wedge \delta A_{\sigma}^{i}\right)
$$


is indeed closed.

Proof: Let us denote by

$$
\mathfrak{d} \Theta_{0}\left(\delta_{1}, \delta_{2}\right)=\delta_{1}\left(\Theta_{0}\left(\delta_{2}\right)\right)-\delta_{2}\left(\Theta_{0}\left(\delta_{1}\right)\right)
$$

the exterior derivative of $\Theta_{0}$. For infinitesimal $S U(2)$ transformations we have

$$
\delta_{\alpha} e=[\alpha, e] \quad \delta_{\alpha} A_{\gamma(\sigma)}=-d_{A_{\gamma(\sigma)}} \alpha
$$

from which it follows:

$$
\begin{aligned}
\mathfrak{d} \Theta_{0}\left(\delta, \delta_{\alpha}\right) & =\int_{H} \delta e^{i} \wedge \delta_{\alpha} e_{i}-\frac{2}{\sigma^{2}-\gamma^{2}} \int_{H}\left(\delta A_{\gamma i} \wedge \delta_{\alpha} A_{\gamma}^{i}-\delta A_{\sigma i} \wedge \delta_{\alpha} A_{\sigma}^{i}\right) \\
& =\int_{H} 2 \delta e^{i} \wedge \epsilon_{i j k} \alpha^{j} e^{k}+\frac{2}{\sigma^{2}-\gamma^{2}} \int_{H}\left(\delta A_{\gamma i} \wedge d_{A_{\gamma}} \alpha^{i}-\delta A_{\sigma i} \wedge d_{A_{\sigma}} \alpha^{i}\right) \\
& =\int_{H} 2 \delta e^{i} \wedge e^{k} \alpha^{j} \epsilon_{i j k}+\frac{2}{\sigma^{2}-\gamma^{2}} \int_{H}\left(\left(d_{A_{\gamma}} \delta A_{\gamma i}\right) \alpha^{i}-\left(d_{A_{\sigma}} \delta A_{\sigma i}\right) \alpha^{i}\right) \\
& =\int_{H}-2 \epsilon_{i k j} \delta e^{i} \wedge e^{k} \alpha^{j}+\frac{2}{\sigma^{2}-\gamma^{2}} \int_{H}\left(\delta F_{i}\left(A_{\gamma}\right) \alpha^{i}-\delta F_{i}\left(A_{\sigma}\right) \alpha^{i}\right) \\
& =\int_{H} \delta\left(-\Sigma^{i}+\frac{2}{\sigma^{2}-\gamma^{2}}\left(F_{i}\left(A_{\gamma}\right)-F_{i}\left(A_{\sigma}\right)\right)\right) \alpha_{i}
\end{aligned}
$$

where in the third line we have integrated by parts. Therefore, from (64), we have that

$$
\mathfrak{d} \Theta_{0}\left(\delta, \delta_{\alpha}\right)=0
$$

For tangent diffeomorphisms on $H$, using Equation (31), we have

$$
\begin{aligned}
\mathfrak{d} \Theta_{0}\left(\delta, \delta_{v}\right) & =\int_{H} 2 \delta e^{i} \wedge \delta_{v} e_{i}-\frac{2}{\sigma^{2}-\gamma^{2}} \int_{H}\left(\delta A_{\gamma i} \wedge \delta_{v} A_{\gamma}^{i}-\delta A_{\sigma i} \wedge \delta_{v} A_{\sigma}^{i}\right) \\
& \left.\left.=\int_{H} 2 v\right\lrcorner \delta e_{i} \wedge d e^{i}+2 \delta d e_{i} \wedge v\right\lrcorner e^{i} \\
& \left.\left.-\frac{2}{\sigma^{2}-\gamma^{2}} \int_{H}\left(\delta \Gamma_{i} \wedge v\right\lrcorner\left(F^{i}\left(A_{\gamma}\right)-F^{i}\left(A_{\sigma}\right)\right)+(\gamma-\sigma) \delta e_{i} \wedge v\right\lrcorner\left(F^{i}\left(A_{\gamma}\right)-F^{i}\left(A_{\sigma}\right)\right)\right) \\
& \left.\left.-\frac{2}{\sigma^{2}-\gamma^{2}} \int_{H}\left(\delta F_{i}\left(A_{\gamma}\right) v\right\lrcorner A_{\gamma}^{i}-\delta F_{i}\left(A_{\sigma}\right) v\right\lrcorner A_{\sigma}^{i}\right) \\
& \left.=\int_{H} 2 \delta\left(d e^{i} \wedge v\right\lrcorner e_{i}\right)-\frac{2}{\sigma^{2}-\gamma^{2}}(\gamma-\sigma) \int_{H} \underbrace{\left.\delta\left(e_{i} \wedge v\right\lrcorner\left(F^{i}\left(A_{\gamma}\right)-F^{i}\left(A_{\sigma}\right)\right)\right)} \\
& \left.\left.-\int_{H}\left(\delta \Gamma_{i} \wedge v\right\lrcorner \Sigma_{i} \wedge v\right\lrcorner \Sigma^{i}\right)=0 \\
& \left.\left.=\int_{H}-2 \delta\left(\epsilon^{i}{ }_{j k} \Gamma^{j} \wedge e^{k} \wedge v\right\lrcorner e_{i}\right)-\int_{H} \delta\left(\Gamma^{i} \wedge v\right\lrcorner \Sigma_{i}\right) \\
& \left.\left.=\int_{H} \delta\left(\Gamma^{i} \wedge v\right\lrcorner \Sigma_{i}\right)-\int_{H} \delta\left(\Gamma^{i} \wedge v\right\lrcorner \Sigma_{i}\right)=0
\end{aligned}
$$

where in addition to integrating by parts and using that $\partial H=0$, we have used Cartan's structure equation (34), the fact that $e^{1}$ is orthogonal to $H$ (that is why the expression below the underbracket is zero), and the identities $A \wedge v\lrcorner B=-v\lrcorner A \wedge B$ valid for any 2-form $A$ and 1-form $B$ on a 2-manifold or any 2-form $A$ and 2-form $B$ on a 3-manifold. 
The previous statement implies that the boundary symplectic form can be rewritten as

$$
\beta \kappa \Omega\left(\delta_{1}, \delta_{2}\right)=\int_{M} \delta_{[1} \Sigma^{i} \wedge \delta_{2]} A_{i}-\frac{1}{\left(\sigma^{2}-\gamma^{2}\right)} \int_{H}\left(\delta_{[1} A_{\gamma i} \wedge \delta_{2]} A_{\gamma}^{i}-\delta_{[1} A_{\sigma i} \wedge \delta_{2]} A_{\sigma}^{i}\right)
$$

From the previous equation we conclude that the boundary term is given by the simplectic structure of two Chern-Simons theories with levels

$$
k_{\gamma}=-k_{\sigma}=\frac{8 \pi}{\left(\sigma^{2}-\gamma^{2}\right) \kappa \beta}
$$

\section{Quantization}

The form of the symplectic structure motivates one to handle the quantization of the bulk and horizon degrees of freedom separately. We first discuss the bulk quantization. As in standard LQG [6-9] one first considers (bulk) Hilbert spaces $\mathscr{H}_{\gamma}^{B}$ defined on a graph $\gamma \subset M$ and then takes the projective limit containing the Hilbert spaces for arbitrary graphs. Along these lines let us first consider $\mathscr{H}_{\gamma}^{B}$ for a fixed graph $\gamma \subset M$ with end points on $H$, denoted $\gamma \cap H$. The quantum operator associated with $\Sigma$ is the flux operator of LQG that can be written as

$$
\epsilon^{c d} \sum_{c d}^{i}(x)=16 \pi G \hbar \beta \sum_{p \in \gamma \cap H} \delta\left(x, x_{p}\right) J_{b}^{i}(p)
$$

where $J_{b}^{i}(p)$ are $S U(2)$ infinitesimal generators, such that $\left[J_{b}^{i}(p), J_{b}^{j}(p)\right]=\epsilon^{i j}{ }_{k} J_{b}^{k}(p)$, acting at each $p \in \gamma \cap H$ and $\gamma \subset M$ is a graph with end points $p$ on $H$ (for more details see [6-9]). The index $b$ in $J_{b}(p)$ is not a space-time index, but simply stands for bulk. Consider a basis of $\mathscr{H}_{\gamma}^{B}$ of eigenstates of both $J(p) \cdot J(p)$ as well as $J^{3}(p)$ for all $p \in \gamma \cap H$ with eigenvalues $\hbar^{2} j_{p}\left(j_{p}+1\right)$ and $\hbar m_{p}$ respectively. These states are spin network states, here denoted $\left|\left\{j_{p}, m_{p}\right\}_{1}^{n} ; \ldots\right\rangle$, where $j_{p}$ and $m_{p}$ are the spins and magnetic numbers labeling the $n$ edges puncturing the horizon at points $x_{p}$ (other labels are left implicit). They are also eigenstates of the horizon area operator $a_{H}$

$$
a_{H}\left|\left\{j_{p}, m_{p}\right\}_{1}^{n} ; \cdots\right\rangle=8 \pi \beta \ell_{p}^{2} \sum_{p=1}^{n} \sqrt{j_{p}\left(j_{p}+1\right)}\left|\left\{j_{p}, m_{p}\right\}_{1}^{n} ; \cdots\right\rangle
$$

Now, following Witten's prescription to quantize the two Chern-Simons theories with punctures [47], we introduce:

$$
\frac{k_{\gamma}}{4 \pi \hbar} F^{i}\left(A_{\gamma}\right)=J_{\gamma}^{i}(p) \quad \frac{k_{\sigma}}{4 \pi \hbar} F^{i}\left(A_{\sigma}\right)=J_{\sigma}^{i}(p)
$$

where

$$
k_{\gamma}=-k_{\sigma}=\frac{8 \pi}{\left(\sigma^{2}-\gamma^{2}\right) \kappa \beta}
$$

are the two levels of the two $S U(2)$ Chern-Simons theories involved in our model. If we do so we can now write the constraints as (recall (64)):

$$
\begin{aligned}
& J_{\gamma}^{i}(p)=\frac{2 \Psi_{2}+\left(\gamma^{2}+c\right)}{\left(\sigma^{2}-\gamma^{2}\right)} J_{b}^{i}(p) \\
& J_{\sigma}^{i}(p)=-\frac{2 \Psi_{2}+\left(\sigma^{2}+c\right)}{\left(\sigma^{2}-\gamma^{2}\right)} J_{b}^{i}(p)
\end{aligned}
$$


So now from (76) we obtain the constraint

$$
D^{i}(p)=J_{b}^{i}(p)+J_{\gamma}^{i}(p)+J_{\sigma}^{i}(p)=0
$$

plus the constraint

$$
C^{i}(p)=J_{\gamma}^{i}(p)-J_{\sigma}^{i}(p)+\frac{2 d+\left(\sigma^{2}+\gamma^{2}\right)}{\left(\sigma^{2}-\gamma^{2}\right)} J_{b}^{i}(p)=0
$$

The constraints $C^{i}(p)=0$ will provide the definition of $d=2 \Psi_{2}+c$ in the quantum theory (see Equation (20)). This point will be clarified at the end of the following subsection. From now on we shall set $\hbar=1$ for notational convenience.

We can quantize the boundary theory following Witten's prescription. In fact, the Hilbert space of the boundary model is that of two Chern-Simons theories associated with a pair of spins $\left(j_{p}^{\gamma}, j_{p}^{\sigma}\right)$ at each puncture. More precisely,

$$
\mathscr{H}_{k}^{C S}\left(j_{1}^{\gamma} \cdots j_{n}^{\gamma}\right) \otimes \mathscr{H}_{k}^{C S}\left(j_{1}^{\sigma} \cdots j_{n}^{\sigma}\right) \subset \operatorname{Inv}\left(j_{1}^{\gamma} \otimes \cdots \otimes j_{n}^{\gamma}\right) \otimes \operatorname{Inv}\left(j_{1}^{\sigma} \otimes \cdots \otimes j_{n}^{\sigma}\right)
$$

The Hilbert space of CS theory with given punctures on the sphere can be thought of as the intertwiner space of the quantum deformation of $S U(2)$ denoted $U_{q}(s u(2))$. The inclusion symbol in the previous expression means that the later space is isomorphic to a subspace of classical $S U(2)$ intertwiner space. This is due to the fact that, in this isomorphism, the spins associated to the $C S$ punctures cannot take all values allowed by the representation theory of $S U(2)$, but are restricted by the cut-off $k / 2$ related to the deformation parameter by $q=\exp \left(\frac{i \pi}{k+2}\right)$ where $k$ is the Chern-Simons level.

The operators associated to $J_{\gamma}^{i}(p)$ and $J_{\sigma}^{i}(p)$, on the other hand, describe the spins of the pair of CS defects at the punctures. They are observables of the boundary system with which the spins $j_{p}^{\gamma}$ and $j_{p}^{\sigma}$ are associated. The theory is topological which means in our case that non-trivial d.o.f. are only present at punctures. With all this we can now impose (77) which, at a single puncture, requires invariance under $S U(2)$ local transformations

$$
\delta J_{A}^{j}=\left[\alpha_{i} D^{i}, J_{A}^{j}\right]=\epsilon_{k}^{i j} \alpha_{i} J_{A}^{k}
$$

where the subscript $A$ here stands for $\gamma, \sigma$, and $b$ respectively. Equivalently, the constraint (77) requires the quantum state to be proportional to the singlet state with zero total $S U(2)$ charge: zero total angular momentum. More precisely, the constraint $D^{i}(p)=0$ simply requires that

$$
\operatorname{Inv}\left(j_{p} \otimes j_{p}^{\gamma} \otimes j_{p}^{\sigma}\right) \neq \emptyset
$$

at each puncture $p$.

\section{Learning from the Restriction to Type I Isolated Horizons}

Let us now show how, within this new approach, we are able to recover the same picture of the spherically symmetric case as studied in [23]. We will show how this will allow us to reduce from two to one the number of free parameters describing the system. For a spherically symmetric IH we can replace in (76) $\Psi_{2}$ and $c$ with their constant classical values: $\Psi_{2}=-\frac{2 \pi}{a_{H}}$ and $c=\frac{2 \pi}{a_{H}}$ (see [23]). If we do so, we obtain the two sets of constraints:

$$
D^{i}(p) \equiv J_{b}^{i}(p)+J_{\gamma}^{i}(p)+J_{\sigma}^{i}(p)=0 \quad C^{i}(p) \equiv J_{\gamma}^{i}(p)-J_{\sigma}^{i}(p)+\alpha J_{b}^{i}(p)=0
$$


where

$$
\alpha \equiv \frac{\left(\sigma^{2}+\gamma^{2}\right)-\frac{4 \pi}{a_{H}}}{\sigma^{2}-\gamma^{2}}
$$

The form of the symplectic structure as in (60) and (63) — together with Equations (74) - implies the commutation relations $\left[J_{\gamma}^{i}(p), J_{\gamma}^{j}(p)\right]=\epsilon^{i j}{ }_{k} J_{\gamma}^{k}(p),\left[J_{\sigma}^{i}(p), J_{\sigma}^{j}(p)\right]=\epsilon^{i j}{ }_{k} J_{\sigma}^{k}(p)$, and $\left[J_{b}^{i}(p), J_{b}^{j}(p)\right]=$ $\epsilon^{i j}{ }_{k} J_{b}^{k}(p)$ from which the constraint algebra that follows is:

$$
\begin{aligned}
& {\left[C^{i}(p), C^{j}\left(p^{\prime}\right)\right]=\epsilon^{i j}{ }_{k}\left(J_{\gamma}^{k}(p)+J_{\sigma}^{k}(p)+\alpha^{2} J_{b}^{k}(p)\right) \delta_{p p^{\prime}}} \\
& {\left[C^{i}(p), D^{j}\left(p^{\prime}\right)\right]=\epsilon^{i j}{ }_{k} C^{k}(p) \delta_{p p^{\prime}}} \\
& {\left[D^{i}(p), D^{j}\left(p^{\prime}\right)\right]=\epsilon^{i j}{ }_{k} D^{k}(p) \delta_{p p^{\prime}}}
\end{aligned}
$$

We can thus see that, by setting $\alpha^{2}=1$ the algebra of constraints closes yielding the simple result

$$
\begin{aligned}
& {\left[C^{i}(p), C^{j}\left(p^{\prime}\right)\right]=\epsilon^{i j}{ }_{k} D^{k}(p) \delta_{p p^{\prime}}} \\
& {\left[C^{i}(p), D^{j}\left(p^{\prime}\right)\right]=\epsilon^{i j}{ }_{k} C^{k}(p) \delta_{p p^{\prime}}} \\
& {\left[D^{i}(p), D^{j}\left(p^{\prime}\right)\right]=\epsilon^{i j}{ }_{k} D^{k}(p) \delta_{p p^{\prime}}}
\end{aligned}
$$

If we introduce the dimensionless parameters $\gamma_{0}$ and $\sigma_{0}$ so that

$$
\gamma=\sqrt{\frac{2 \pi}{a_{H}}} \gamma_{0}, \quad \sigma=\sqrt{\frac{2 \pi}{a_{H}}} \sigma_{0}
$$

then the previous analysis implies that we can impose spherical symmetry strongly if and only if $\left(\sigma_{0}^{2}+\gamma_{0}^{2}\right) \pm\left(\sigma_{0}^{2}-\gamma_{0}^{2}\right)=2$. A possible solution (for the plus branch, or $\alpha=-1$ ) is for instance $\sigma_{0}=1$ and $\gamma_{0}$ arbitrary, for which the level (75) becomes:

$$
k=\frac{a_{H}}{\frac{\kappa \beta}{4}\left(1-\gamma_{0}^{2}\right)}
$$

which exactly matches the value found in [23] (see section VII) for Type I isolated horizons. Moreover, defining:

$$
C_{ \pm}^{i}(p) \equiv \frac{D^{i}(p) \pm C^{i}(p)}{2} \quad \Rightarrow \quad C_{+}^{i}(p)=J_{b}^{i}(p)+J_{\gamma}^{i}(p) \quad C_{-}^{i}(p)=J_{\sigma}^{i}(p)
$$

the algebra (85)-(87) becomes:

$$
\begin{aligned}
& {\left[C_{ \pm}^{i}(p), C_{ \pm}^{j}\left(p^{\prime}\right)\right]=\epsilon_{k}^{i j} C_{ \pm}^{k}(p) \delta_{p p^{\prime}}} \\
& {\left[C_{ \pm}^{i}(p), C_{\mp}^{j}\left(p^{\prime}\right)\right]=0}
\end{aligned}
$$

and therefore we can impose $C_{ \pm}^{i}=0$ strongly which boils down to setting $j_{p}^{\sigma}=0$ and $j_{p}^{\gamma}=j_{p}$ on the boundary spins. In this way the Hilbert space of generic static isolated horizons $\mathscr{H}_{k}^{C S}\left(j_{1}^{\gamma} \cdots j_{n}^{\gamma}\right) \otimes$ $\mathscr{H}_{k}^{C S}\left(j_{1}^{\sigma} \cdots j_{n}^{\sigma}\right)$ (restricted only by the condition (80)) reduces for Type I isolated horizons to $\mathscr{H}^{C S}\left(j_{1} \cdots j_{n}\right) \subset \operatorname{Inv}\left(j_{1} \otimes \cdots \otimes j_{n}\right)$ in complete agreement with [23]. This correspondence works also at the classical level. We have seen that spherical symmetry implies $C_{-}=0$ which, according to Equation (74), requires

$$
F\left(A_{\sigma}\right)=0
$$


As the horizon $H$ is simply connected, this implies that $A_{\sigma}=g d g^{-1}$, i.e., pure gauge. Therefore, the non-trivial degrees of freedom of the Type I isolated horizon are described by a single Chern-Simons theory with connection $A_{\gamma}$ and constraint $C_{+}=0$ equivalent to

$$
\frac{k}{4 \pi} F\left(A_{\gamma}\right)=\Sigma^{i}
$$

in complete classical correspondence with the treatment of [23]. Therefore, the requirement that spherical symmetry can be imposed strongly reduces the two parameter family of models of a distorted horizon to a one-parameter one. This one-parameter ambiguity is also present in the spherical isolated horizon case (yet it does not affect the entropy calculation).

Before ending this section let us make two important points.

The first point concerns the constraint structure at places where $J_{b}^{2}=0$, i.e., where there is no puncture. We have already argued that at these points $J_{\sigma}^{i}(p)=0=J_{\gamma}^{i}(p)$ above, but it is instructive to briefly revisit the point here. When $J_{b}^{2}(p)=0$ the constraints reduce to

$$
\begin{aligned}
& C^{i}(p)=J_{\sigma}^{i}(p)+J_{\gamma}^{i}(p)=0 \\
& D^{i}(p)=J_{\gamma}^{i}(p)-J_{\sigma}^{i}(p)=0
\end{aligned}
$$

with $\left[C^{i}(p), D^{j}\left(p^{\prime}\right)\right]=\epsilon^{i j k} D_{k}(p) \delta_{p p^{\prime}}$. There are 6 first class constraints equivalent to $J_{\sigma}^{i}(p)=0=J_{\gamma}^{i}(p)$ and hence no degrees of freedom at these points.

At points where $J_{b}^{2}(p) \neq 0$ the situation is more complex. In addition to the constraints $D^{i}(p)=0$ one has to impose the three additional constraints $C^{i}(p)=0$ that take the form (78) in the arbitrarily distorted case. The problem is that, as shown above (see Equation (82)), the constraint algebra does not close in the present case. Therefore, since the set of constraints $D^{i}(p), C^{i}(p)$ is no more first class in the generic distorted case, imposing the six constraints strongly is a far too strong requirement that risks to kill relevant physical degrees of freedom. The reason is that, in addition to the constraints $D^{i}(p)=0$ and $C^{i}(p)=0$, one is imposing an infinite tower of constraints stemming from arbitrary order commutators of the original ones: we will see that the only solutions to these are indeed spherical configurations.

Fortunately there is a natural way of imposing the constraints weakly. First we notice that-using the closure constraint $D^{i}(p)=0$, which we do impose strongly as they are first class- the constraint $C^{i}(p)$ can be written in the following form

$$
C^{i}(p) \approx J_{\gamma}^{i}(p)-J_{\sigma}^{i}(p)-\alpha\left(J_{\sigma}^{i}(p)+J_{\gamma}^{i}(p)\right)=0
$$

If we interpret for a moment the previous constraint classically we see that it implies that the vectors $J_{\gamma}^{i}(p), J_{\sigma}^{i}(p)$, and (through $\left.D^{i}(p)=0\right) J_{b}^{i}(p)$ are parallel. This is exactly the role of the constraint $C(p)^{i}=0$, as it follows directly from the classical equations (64) where one explicitly sees that all sources of curvature are proportional to the single field $\Sigma^{i}$.

In order to define our strategy for a relaxation of the constraint $C^{i}(p)=0$, it is particularly clarifying to take the perspective of the master constraint technology developed by Thiemann et al. [52,53]. If we chose to impose the equivalent set of (now commuting) constraints given by gauge constraint $D^{i}(p)=0$ - which implements the $S U(2)$ gauge symmetry at each of the punctures-in addition to the 
master constraint $C^{2}(p)=0$ one would find that the only states in the kernel of $C^{i}(p)$ are spherically symmetric states. Namely, the latter takes the following explicit form:

$$
C^{2}(p)=2 J_{\gamma}^{2}(p)+2 J_{\sigma}^{2}(p)-J_{b}^{2}(p)+2 \alpha\left(J_{\sigma}^{2}(p)-J_{\gamma}^{2}(p)\right)+\alpha^{2} J_{b}^{2}(p)
$$

with

$$
\alpha=\frac{\frac{a_{H}}{\pi} d+\left(1+\gamma_{0}^{2}\right)}{\left(1-\gamma_{0}^{2}\right)}
$$

The previous operator is positive definite and the condition that it exists $\alpha$ such that (96) vanishes is given by the following restriction (stemming from the resolvent of the previous quadratic equation)

$$
\begin{aligned}
& \left(J_{\sigma}^{2}(p)-J_{\gamma}^{2}(p)\right)^{2}-J_{b}^{2}(p)\left(2 J_{\gamma}^{2}(p)+2 J_{\sigma}^{2}(p)-J_{b}^{2}(p)\right)=0 \\
& J_{\sigma}^{2}(p) J_{\gamma}^{2}(p)-\left(J(p)_{\gamma} \cdot J(p)_{\sigma}\right)^{2}=0
\end{aligned}
$$

where we have written it in two equivalent forms. In the last line we see that the condition is equivalent to the vanishing of the quantum angle $\varphi_{\sigma \gamma}$ between $J_{\gamma}^{i}(p)$ and $J_{\sigma}^{i}(p)$, whose cosine takes the form

$$
\cos \left(\varphi_{\gamma \sigma}\right)=\frac{J(p)_{\gamma} \cdot J(p)_{\sigma}}{\sqrt{J(p)_{\gamma}^{2} J(p)_{\sigma}^{2}}}
$$

The only strict solutions of that constraint are $J_{\sigma}^{i}(p)=0$ or $J_{\gamma}^{i}(p)=0$ which give $\alpha= \pm 1$ and hence spherically symmetric states only. We have to relax the previous constraint and impose it weakly. We will therefore require the previous equation to hold only in the large spin limit. In that case it is immediate to see that the constraint implies the simple condition

$$
j_{b}=j^{\sigma}+j^{\gamma}
$$

As shown by Major in [54] the minimal angle goes as $1 / \sqrt{j_{b}}$. All this implies that it is consistent to take

$$
\alpha \equiv \frac{J_{\sigma}^{2}(p)-J_{\gamma}^{2}(p)}{J_{b}^{2}(p)}
$$

and hence we take

$$
d \equiv 2 \Psi_{2}+c=\frac{\pi}{a_{H}} \frac{\left(1-\gamma_{0}^{2}\right)\left(J_{\sigma}^{2}(p)-J_{\gamma}^{2}(p)\right)-\left(1+\gamma_{0}^{2}\right) J_{b}^{2}(p)}{J_{b}^{2}(p)}
$$

in the quantum theory as well. In other words the operator associated to the latter quantity make sense and has no fluctuations in the Hilbert space of the distorted horizon. Notice that Equation (101) would follow from the strong imposition of the component $C(p) \cdot J_{b}(p)$ of $C^{i}(p)$ which together with $D^{i}(p)$ form a set of commuting constraints. Notice as well that $\alpha$ defined in (101) commutes with all the observables in the boundary system! Thus, the quantity $d=2 \Psi_{2}+c$ remains 'classical' in this sense in agreement with the assumptions used for the construction of the phase space of our system.

Remark 1: There is another branch of approximate solutions (in the large spin limit) to the condition (98) corresponding to $j_{b}=\left|j_{\sigma}-j_{\gamma}\right|$; however, these correspond to antiparallel configurations which are ruled out by the form of the original constraints. See for instance (64). 
Remark 2: There is a strict analogy between the way we have imposed the constraint $C^{i}(p)=0$ in this section and the way the simplicity constraints are imposed in the EPRL-FK model $[55,56]$. Observe first that Equation (95) has the very same form of the linear simplicity constraints of the EPRL-FK models where the role of the Immirzi parameter is here played by $\alpha$. Notice also that if we take $j_{\gamma}=(1-\alpha) j / 2$ and $j_{\sigma}=(1+\alpha) j / 2$ then this solves Equation (100) and can be checked to be consistent with alpha as given (101). With this then one can check that for an admissible state $|\psi\rangle$ one has

$$
C^{2}|\psi\rangle=\hbar^{2}\left(1-\alpha^{2}\right) j|\psi\rangle
$$

which vanishes in the (semiclassical) limit $\hbar \rightarrow 0, j \rightarrow \infty$ with $\hbar j$ kept constant. Moreover, using the results of [57] one has that

$$
\left\langle\phi\left|C^{i}\right| \psi\right\rangle=0
$$

for arbitrary pairs of admissible states. In other words, the constraint $C^{i}$ are satisfied strongly in the semiclassical limit, and weakly in the sense of matrix elements in general.

\section{Entropy Calculation}

From now on we fix the value of $\sigma_{0}$ in the distorted case according to the analysis preformed in Section 5.1, i.e., we set $\sigma_{0}=1$ and keep $\gamma_{0}$ as the only free parameter. With this preferred choice of parameters Equation (81) now becomes:

$$
\begin{aligned}
& D^{i}(p) \equiv J_{b}^{i}(p)+J_{\gamma}^{i}(p)+J_{\sigma}^{i}(p)=0 \\
& J_{\gamma}^{2}(p)-J_{\sigma}^{2}(p)+\frac{\frac{a_{H}}{\pi}\left(2 \Psi_{2}+c\right)+\left(1+\gamma_{0}^{2}\right)}{\left(1-\gamma_{0}^{2}\right)} J_{b}^{2}(p)=0
\end{aligned}
$$

where, we remind, $\Psi_{2}$ is the Weyl tensor component defined by $\Psi_{2}=C_{a b c d} \ell^{a} m^{b} \bar{m}^{c} n^{d}$, with $C_{a b c d}$ the Weyl tensor and $\ell, n, m$ the null tetrad defined in Statement 1 of Section 3, which is simply related to the local scalar curvature of $H$. The quantity $c$ is the function relating extrinsic and intrinsic curvature through relation (12). Both $\Psi_{2}$ and $c$ are functions of the horizon points, while Lie dragged along the null generators $\ell^{a}$.

From (103) we get

$$
d=2 \Psi_{2}+c=\frac{\pi}{a_{H}} \frac{\left(1-\gamma_{0}^{2}\right)\left(J_{\sigma}^{2}(p)-J_{\gamma}^{2}(p)\right)-\left(1+\gamma_{0}^{2}\right) J_{b}^{2}(p)}{J_{b}^{2}(p)}
$$

The previous equation represents a well-defined expression for an operator encoding the degrees of freedom of distortion; its eigenvalues are determined by the spins associated to the bulk and horizon punctures and they characterize the distorted configurations which will contribute to the entropy calculation. More precisely, the sum over the bulk and horizon spins performed (see below) in the states counting corresponds to the sum over the allowed distorted configurations of the model (see remark at the end of Section 3). In this sense, we can trace back the horizon entropy to the counting of the boundary geometry degrees of freedom.

Spherically symmetric states correspond to the eigenvalue $-2 \pi / a_{H}$ of the operator $2 \Psi_{2}+c$. The deviation from spherical symmetry can be encoded in

$$
\Delta \equiv d+\frac{2 \pi}{a_{H}}=\frac{\pi}{a_{H}} \frac{\left(1-\gamma_{0}^{2}\right)\left(J_{\sigma}^{2}(p)-J_{\gamma}^{2}(p)+J_{b}^{2}(p)\right)}{J_{b}^{2}(p)}
$$


in fact, the spherically symmetric configurations correspond to set-see Equation (89) $-j^{\sigma}=0$ and $j_{b}=j^{\gamma}$, giving the zero eigenvalue of $\Delta$.

We can now ask wether including all kind of distortions-allowing $j_{p}^{\gamma}, j_{p}^{\sigma}$ to go all the way up to the cut off $k / 2$ - preserves the area law for the IH entropy.

\subsection{The Usual Paradigm}

In this first counting we take the usual approach where the Chern-Simons level grows with $a_{H}$ according to (88). Consequently we neglect the quantum corrections coming from the (quantum group) closure constraint in consistency with the large $k$ limit, which is equivalent to the large area asymptotics (i.e., the thermodynamic limit). We will show that the inclusion of distortion does not violate the area law but simply amounts to fixing the Immirzi parameter to value that is larger than the one found in the spherically symmetric case.

In order to compute the number of states contained in the Hilbert space (79) we are now going to use the counting techniques of [49]. For a generic configuration of spins associated to the punctures on the horizon we denote $s\left(j^{\gamma}, j^{\sigma}\right)$ the occupation numbers, i.e., the number of punctures labeled by internal spins $j^{\gamma}$ and $j^{\sigma}$ respectively. One can easily see that, keeping in mind the constraints $j_{p}^{\gamma}, j_{p}^{\sigma} \leq k / 2$, for a given configuration $\left\{s\left(j^{\gamma}, j^{\sigma}\right)\right\}$, the total number of quantum states reads

$$
d\left(\left\{s\left(j^{\gamma}, j^{\sigma}\right)\right\}\right)=\frac{\left[\sum_{j^{\gamma}, j^{\sigma}} s\left(j^{\gamma}, j^{\sigma}\right)\right] !}{\prod_{j^{\gamma}, j^{\sigma}} s\left(j^{\gamma}, j^{\sigma}\right) !} \prod_{j^{\gamma}, j^{\sigma}=0}^{\frac{k}{2}}\left(\left(2 j^{\gamma}+1\right)\left(2 j^{\sigma}+1\right)\right)^{s\left(j^{\gamma}, j^{\sigma}\right)}
$$

where the combinatorial factor comes from the fact that the punctures are considered distinguishable. To obtain the total number of states one should then sum over all possible configuration $\left\{s\left(j^{\gamma}, j^{\sigma}\right)\right\}$. Following [49], we estimate the sum by maximizing $\ln d\left(\left\{s\left(j^{\gamma}, j^{\sigma}\right)\right\}\right)$ by varying $s\left(j^{\gamma}, j^{\sigma}\right)$ subject to the area constraint

$$
\sum_{j^{\gamma}, j^{\sigma}} s\left(j^{\gamma}, j^{\sigma}\right) \sqrt{\left(j^{\gamma}+j^{\sigma}\right)\left(j^{\gamma}+j^{\sigma}+1\right)}=\frac{a_{H}}{8 \pi \beta \ell_{p}^{2}}
$$

where the above form of the area constraint follows from the area spectrum (73) in LQG and the condition (100). In the variation we assume that $s\left(j^{\gamma}, j^{\sigma}\right)>>1$ for each $j^{\gamma}, j^{\sigma}$ and only such configurations dominate the counting. Introducing the Lagrange multiplier $\lambda$, the variational equation $\delta \ln d\left(\left\{s\left(j^{\gamma}, j^{\sigma}\right)\right\}\right)=\lambda \delta a_{H}$ gives

$$
\frac{s\left(j^{\gamma}, j^{\sigma}\right)}{\sum_{j^{\gamma}, j^{\sigma}} s\left(j^{\gamma}, j^{\sigma}\right)}=\left(2 j^{\gamma}+1\right)\left(2 j^{\sigma}+1\right) e^{-\lambda 8 \pi \beta \ell_{p}^{2} \sqrt{\left(j^{\gamma}+j^{\sigma}\right)\left(j^{\gamma}+j^{\sigma}+1\right)}}
$$

From the previous relation we obtain $\lambda \equiv \lambda_{0} /\left(8 \pi \beta \ell_{p}^{2}\right)$ as a solution of

$$
1=\sum_{j^{\gamma}, j^{\sigma}=1 / 2}^{k / 2}\left(2 j^{\gamma}+1\right)\left(2 j^{\sigma}+1\right) e^{-\lambda_{0} \sqrt{\left(j^{\gamma}+j^{\sigma}\right)\left(j^{\gamma}+j^{\sigma}+1\right)}}
$$

obtained by summing Equation (108) over $j^{\gamma}, j^{\sigma}$. 
To estimate the leading order in the entropy associated to the total number of quantum states for all configurations $\left\{s\left(j^{\gamma}, j^{\sigma}\right)\right\}$ we evaluate the logarithm of (106) at the dominant configuration (108), which we denote $\bar{s}\left(j^{\gamma}, j^{\sigma}\right)$, namely

$$
\begin{aligned}
\log d_{\left\{\bar{s}\left(j^{\gamma}, j^{\sigma}\right)\right\}} & \approx \sum_{j^{\gamma}, j^{\sigma}} s\left(j^{\gamma}, j^{\sigma}\right)\left(\log \sum_{j^{\gamma}, j^{\sigma}} s\left(j^{\gamma}, j^{\sigma}\right)-\log s\left(j^{\gamma}, j^{\sigma}\right)+\log \left(2 j^{\gamma}+1\right)\left(2 j^{\sigma}+1\right)\right) \\
& =\sum_{j^{\gamma}, j^{\sigma}} s\left(j^{\gamma}, j^{\sigma}\right)\left(\lambda 8 \pi \beta \ell_{p}^{2} \sqrt{\left(j^{\gamma}+j^{\sigma}\right)\left(j^{\gamma}+j^{\sigma}+1\right)}\right)=\lambda a_{H}
\end{aligned}
$$

where in the first line we have used Stirling's approximation. We conclude

$$
S \approx \log d_{\left\{\bar{s}\left(j^{\gamma}, j^{\sigma}\right)\right\}}=\lambda a_{H}+\mathscr{O}\left(\log a_{H}\right)
$$

where $\lambda=\lambda_{0} /\left(8 \pi \beta \ell_{p}^{2}\right)$ for $\lambda_{0}$ a solution of Equation (109): numerically $\lambda_{0}=2.1589 \ldots$ All this implies that the area law is recovered for $\beta=0.343599 \ldots$ instead of the value obtained in the spherically symmetric treatment $\beta_{\text {sph }}=0.274067 \ldots$.

\subsection{A Paradigm Shift: An $a_{H}$-Independent Effective Theory}

We have seen that, if one follows the standard paradigm, the inclusion of distortion in the statistical ensemble produces an entropy that grows in agreement with the area law as long as the Immirzi parameter $\beta$ is fixed to a given numerical value. In the present treatment though, there is an additional ambiguity controlled by the parameter $\gamma_{0}$ (as stressed at the beginning of this section). Equation (88) shows that this ambiguity can be encoded in the value of the level $k$. Thus, the paradigm shift that we propose consists of taking $k \in \mathbb{N}$ as an arbitrary input in the construction of the effective theory describing the phase space of generic IH: we can make $k$ area independent through the free parameter $\gamma_{0}$ by reabsorbing in it the dependence on $a_{H}$. More specifically, assuming $\gamma_{0}^{2}=\left(1-n \kappa \beta /\left(4 a_{H}\right)\right)$, where $n \in \mathbb{N}$, from Equation (88) we see that the level $k$ is now free to take any integer value $n$.

The advantages of this is that, on the one hand, it gives a theory which is independent of any macroscopic parameter-eliminating in this simple way the tension present in the old treatment associated to the natural question: why should the fundamental quantum excitations responsible for black hole entropy know about the macroscopic area of the black hole? —on the other hand, compatibility with the area law will (as shown below) only fix the relationship between the level $k$ and the Immirzi parameter $\beta$; thus no longer constraining the latter to a specific numerical value.

For simplicity let us take the simplest case $k=1$; the general case is considered at the end of the subsection. If we fix $k=1$ then the spins at internal punctures can take only the values $j^{\gamma}, j^{\sigma}=0,1 / 2$. Therefore, there are only three possible non trivial occupation numbers:

$$
A \equiv s(0,1 / 2), \quad B \equiv s(1 / 2,0), \quad C \equiv s(1 / 2,1 / 2)
$$

where the first two cases correspond to spherically symmetric configurations. The total number of states for $A, B$, and $C$ given is

$$
\begin{aligned}
d & =\frac{(A+B+C) !}{A ! B ! C !} \operatorname{dim}\left[\mathscr{H}_{1}^{C S}(\underbrace{1 / 2 \cdots 1}_{B+C \text { times }})\right] \operatorname{dim}\left[\mathscr{H}_{1}^{C S}(\underbrace{1 / 2 \cdots 1 / 2}_{A+C \text { times }})\right] \\
& =\frac{(A+B+C) !}{A ! B ! C !}
\end{aligned}
$$


since, for $k=1$, the dimension of the two $C S$ Hilbert spaces is 1. Extremizing this number with the constraint $a_{H}=\left[(A+B) a_{1 / 2}+C a_{1}\right]$ yields the following equations

$$
\frac{A}{A+B+C}=e^{-\lambda a_{1 / 2}}, \quad \frac{B}{A+B+C}=e^{-\lambda a_{1 / 2}}, \quad \frac{C}{A+B+C}=e^{-\lambda a_{1}}
$$

from which it follows the condition $1=2 e^{-\lambda a_{1 / 2}}+e^{-\lambda a_{1}}$ and a leading order entropy $S=\lambda a_{H}+$ $\mathscr{O}\left(\log \left(a_{H}\right)\right)$. All this implies that we can get exactly Hawking area law

$$
S=\frac{a_{H}}{4 \ell_{p}^{2}}+\mathscr{O}\left(\log \left(a_{H}\right)\right)
$$

if $k=1$ and the Immirzi parameter satisfies:

$$
1=2 e^{-\pi \beta \sqrt{3}}+e^{-2 \pi \beta \sqrt{2}}
$$

The previous renormalization condition is very simple and corresponds to an area independent invariant Chern-Simons level $k=1$. We expect a whole tower of area independent effective theories whose symplectic structure is given by

$$
\Omega_{H}\left(\delta_{1}, \delta_{2}\right)=\frac{k}{8 \pi} \int_{H} \delta_{[1} A_{\gamma}^{i} \wedge \delta_{2]} A_{\gamma i}-\frac{k}{8 \pi} \int_{H} \delta_{[1} A_{\sigma}^{i} \wedge \delta_{2]} A_{\sigma i}
$$

with $k \in \mathbb{N}$ some fixed integer, and constraints

$$
\frac{k}{4 \pi} F^{i}\left(A_{\gamma}\right)=J_{\gamma}^{i}, \quad \frac{k}{4 \pi} F^{i}\left(A_{\sigma}\right)=-J_{\sigma}^{i}
$$

with

$$
J_{\gamma}^{i}+J_{\sigma}^{i}+J_{b}^{i}=0
$$

when $J_{b}^{2} \neq 0$, while $J_{\gamma}^{i}=0$ and $J_{\sigma}^{i}=0$ when $J_{b}^{2}=0$. Provided an appropriate modifications of the renormalization condition (115) for $\beta$ (see below), such effective theory for the horizon degrees of freedom yields $S=a_{H} / 4 \ell_{p}^{2}$ to leading order! The effective theory is independent of the macroscopic parameter $a_{H}$, i.e., the effective model introduced here is one and the same for all $\mathrm{IH}$ and need not to be tuned to the particular value of a parameter that is supposed to be fixed only macroscopically. For completeness we give the expression of the operator (105) controlling the deviations from spherical symmetry which now becomes

$$
\Delta=\frac{1}{8 \ell_{p}^{2} \beta k} \frac{\left(J_{\sigma}^{2}(p)-J_{\gamma}^{2}(p)+J_{b}^{2}(p)\right)}{J_{b}^{2}(p)}
$$

and is independent of macroscopic parameters.

Let us now consider the general case. To explicitly derive the relation that $\beta$ has to satisfy for a generic (area independent) value of the level $k$ such that the area law is recovered we need to use the general formula for the dimension of the Hilbert space $\mathscr{H}_{k}{ }^{C S}\left(j_{1} \cdots j_{n}\right)$, namely $([24-31,59])$

$$
\operatorname{dim}\left[\mathscr{H}_{k}^{C S}\left(j_{1} \cdots j_{n}\right)\right]=\frac{2}{k+2} \sum_{\ell}^{\frac{k}{2}} \sin ^{2}\left(\frac{\pi(2 \ell+1)}{k+2}\right) \prod_{i=1}^{n}\left(\frac{\sin \left(\frac{\pi(2 \ell+1)\left(2 j_{i}+1\right)}{k+2}\right)}{\sin \left(\frac{\pi(2 \ell+1)}{k+2}\right)}\right)
$$


from which we can get

$$
d\left(\left\{s\left(j^{\gamma}, j^{\sigma}\right)\right\}\right)=\frac{\left[\sum_{j^{\gamma}, j^{\sigma}} s\left(j^{\gamma}, j^{\sigma}\right)\right] !}{\prod_{j^{\gamma}, j^{\sigma}} s\left(j^{\gamma}, j^{\sigma}\right) !} N\left(\left\{s\left(j^{\gamma}, j^{\sigma}\right)\right\}\right)
$$

where

$$
\begin{aligned}
N\left(\left\{s\left(j^{\gamma}, j^{\sigma}\right)\right\}\right)= & \frac{4}{(k+2)^{2}} \sum_{\ell, q=0}^{\frac{k}{2}} \sin ^{2}\left(\frac{\pi(2 \ell+1)}{k+2}\right) \sin ^{2}\left(\frac{\pi(2 q+1)}{k+2}\right) \\
& \cdot \prod_{j^{\gamma} j^{\sigma}}\left(\frac{\sin \left(\frac{\pi(2 \ell+1)\left(2 j^{\gamma}+1\right)}{k+2}\right)}{\sin \left(\frac{\pi(2 \ell+1)}{k+2}\right)}\right)^{s\left(j^{\gamma}\right)}\left(\frac{\sin \left(\frac{\pi(2 \ell+1)\left(2 j^{\sigma}+1\right)}{k+2}\right)}{\sin \left(\frac{\pi(2 q+1)}{k+2}\right)}\right)^{s\left(j^{\sigma}\right)}
\end{aligned}
$$

and we have defined $s\left(j^{\gamma}\right) \equiv \sum_{j^{\sigma}} s\left(j^{\gamma}, j^{\sigma}\right)$, and $s\left(j^{\sigma}\right) \equiv \sum_{j^{\gamma}} s\left(j^{\gamma}, j^{\sigma}\right)$. Assuming that

$$
\sum_{j^{\gamma}, j^{\sigma}=0}^{k / 2} s\left(j^{\gamma}, j^{\sigma}\right) \frac{\delta \log \left[N\left(\left\{s\left(j^{\gamma}, j^{\sigma}\right)\right\}\right)\right]}{\delta s\left(j^{\gamma}, j^{\sigma}\right)}-\log \left[N\left(\left\{s\left(j^{\gamma}, j^{\sigma}\right)\right\}\right)\right]=\mathscr{O}\left(\log \left(a_{H}\right)\right)
$$

we obtain that $\beta$ has to satisfy

$$
1=\sum_{j^{\gamma}, j^{\sigma}=0}^{k / 2} \exp \left(\frac{1}{N\left(\left\{s\left(j^{\gamma}, j^{\sigma}\right)\right\}\right)} \frac{\delta N\left(\left\{s\left(j^{\gamma}, j^{\sigma}\right)\right\}\right)}{\delta s\left(j^{\gamma}, j^{\sigma}\right)}\right) \exp \left(-2 \pi \beta \sqrt{\left(j^{\gamma}+j^{\sigma}\right)\left(j^{\gamma}+j^{\sigma}+1\right)}\right)
$$

The previous expression encodes the relationship between $k$ and $\beta_{k}$ dictated by the validity of the area law. Analytic information could be extracted from it by the usual approximation methods or it could be used as the basis for numerical computations. Some exact as well as qualitative information in shown in Figure 2.

Figure 2. The value of the Immirzi parameter $\beta_{k}$ as a function of $k \in \mathbb{N}$ for the first few integers. The value $\beta_{1}=0.172217 \ldots$ is exact as well as the asymptotic value $\beta_{\infty}=0.343599 \ldots$. The other points have been computed using (109) which is only valid in the large $k$ limit.

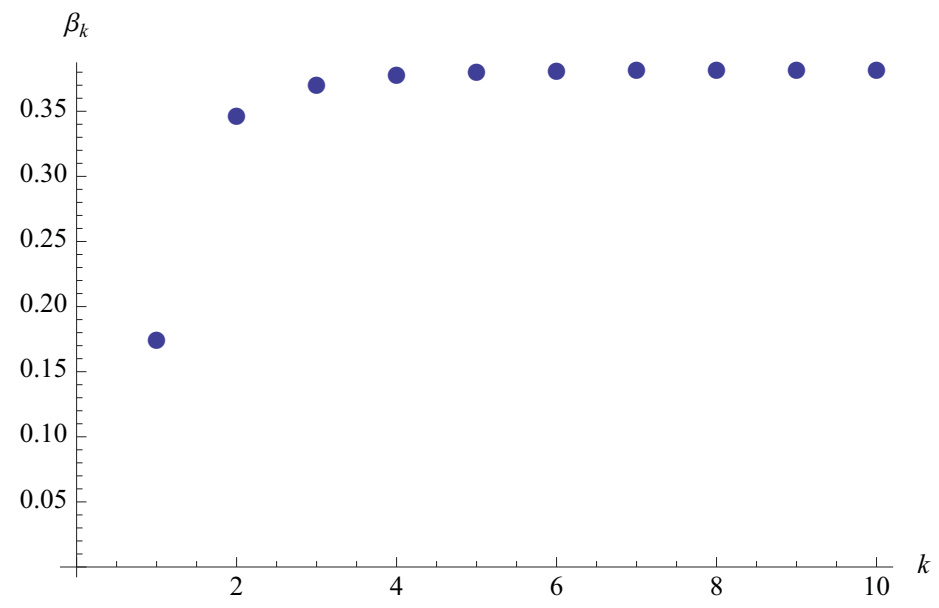


Remark: The presence of an analog ambiguity, in the spherically symmetric case, to the one encoded here in the parameter $\gamma_{0}$ has been already emphasized in [23]. In the full theory such ambiguity is also present [58] but the requirement of the introduction of a scale (such as the $\mathrm{BH}$ area here) at the classical level makes it less natural.

\section{Conclusions}

The main results of this paper can be organized in three parts. In the first part we have shown that it is possible to describe the classical phase space of distorted isolated horizons without the need to invoke the notion of any Type I structure as it was generally done in previous work [34,35]. Our treatment does not require any symmetry assumption treating (static) distorted isolated horizons of type I, II or III on equal footing. Non-static horizons are more complicated systems as our analysis shows due to the breaking of diffeomorphism invariance at the boundary. As we explain in Section 4.4 the breaking of diffeomorphism invariance is only a problem from the point of view of the quantization in the context of loop quantum gravity, as this gauge symmetry breaking is in manifest contradiction to the gauge invariance of the bulk theory. We propose a way to restore diffeomorphism invariance by the enlargement of the kinematical phase space. This strategy is certainly viable, however a clear-cut interpretation of the new system as well as its quantization requires further study. It is important to point out that, as the stationary Kerr-Newman black hole is a non-static isolated horizon, further insights on the issues here discussed are necessary for a complete understanding of the nature of the black hole entropy calculation including rotating horizons.

In the case of static isolated horizons we show that the classical system can be described in terms of certain connection variables. We show that there is a two-parameter family of such variables in terms of which the pre-symplectic structure of the system acquires a boundary term corresponding to the sum of two (closely related) $S U(2)$ Chern-Simons theories. In addition to the pre-symplectic form with its boundary terms, there are constraints that relate the bulk and boundary fields at the isolated horizon and ensure the validity of the boundary condition. The free parameters describing the classically equivalent formulations are the analog of the Immirzi parameter in the full theory in the sense that, as in the latter case, they appear when one changes from the one-form variable phase space parametrization-see Equation (60) — to the connection parametrization — see Equation (63) — that makes the quantization in loop variables more natural. Remarkably, the ambiguity can be entirely encoded in the value of the level of the Chern-Simons theories $k \in \mathbb{N}$ : there is an integer worthy of theories as it becomes clear when rewriting (63) as in Equations (116) with sources as in (117). The conservation of the presymplectic structure proven in Section 4 goes though without making reference to $a_{H}$ and $\gamma_{0}$.

In the second part of the paper we study the quantization of the static isolated horizon phase space. The quantization can be defined along similar lines as the one followed in the spherically symmetric case. However, subtleties arise in the imposition of the quantum boundary conditions. In particular we observe in Section 5.1 that the (boundary) constraint algebra does not close in general. We use this fact to reduce the two-parameter family of classically equivalent formulations to a one-parameter family by requiring that the constraint algebra closes in the case of Type I (i.e., spherically symmetric) isolated horizons. Remarkably, this yields a quantization that is precisely equivalent to the one presented in [22,23]. For distorted isolated horizons this implies that one can impose all the boundary constraints only weakly: 
there is a natural way to impose them in the large spin limit by restricting to those configurations that minimize the 'angle' between the Chern-Simons defects.

We compute the entropy from the quantum theory by counting states in an ensemble containing distorted horizons of a given macroscopic area $a_{H}$ and observe that the inclusion of distortion (in the standard treatment) does not violate the area law, but simply slightly increases the numerical value the Immirzi parameter has to be fixed to. In this regard, it is interesting to notice that this regulating effect of the quantum group which somehow encodes the distortion structure is a consequence of the proper way we have imposed the constraints. In fact, without the restriction (100), i.e., allowing every angle between the two sets of punctures on the horizon, it can be shown that the entropy would grow as $a_{H} \log \left(a_{H}\right)$ in violation of the area law.

In the third part (Section 6.2) we show that the $S U(2)$ treatment allows for a simple paradigm shift where instead of tuning the Immirzi parameter in order to get agreement with Hawkings formula - the usual approach followed in the standard literature-we take the level of the Chern-Simons theory as an independent effective parameter (independent of the area $a_{H}$ ). The advantages of such treatment are multiple. On the one hand the effective theory so defined is universal: independent of any macroscopic parameter such as the area $a_{H}$, i.e., the same for all isolated horizons. On the other hand, from this new perspective one gets agreement with Hawking area law without the need of fixing the Immirzi parameter to a single particular value as there is a integers-worth of possible values of $\beta_{k}$ satisfying the relation (123) for $k \in \mathbb{N}$. From this viewpoint the semiclassical consistency with the Hawking effect is not viewed as a restriction on a fundamental constant in LQG (the Immirzi parameter) but rather as providing insights of a more fundamental (dynamical) description underlying the necessary relationship — manifested here by the admissible effective descriptions labeled by $\left(k, \beta_{k}\right)$ [60]—between the LQG in the bulk and the $S U(2)$ Chern-Simons theory on the boundary. None of two characteristic constants of the bulk theory (Immirzi parameter) and of the boundary theory (CS level) is more important than the other in this model: only a given relationship among the two is required in order to recover the Bekenstein-Hawking formula for black holes entropy [61]. The range of physically suitable values of $\beta_{k}$ is illustrated in Figure 2.

Moreover, the model presented here allows for counting states corresponding to generic isolated horizons, eliminating in this way a puzzling physical restriction imposed in the entropy count of Type I horizons.

It is important to stress that the possibility of taking the Chern-Simons level as an independent parameter is a feature of the $S U(2)$ analysis due to the appearance of a one-parameter freedom lacking in the $U(1)$ case. In particular, the view proposed in Section 6.2 could have been taken as well in the definition of the Type I effective theory leading to the entropy calculation of [23]. If such would have been the view then, only a relationship between the Immirzi parameter and the Chern-Simons level would have been required in order to satisfy the area law in this symmetry reduced case as well. 


\section{Acknowledgements}

We thank Eugenio Bianchi, Jonathan Engle, Laurent Freidel, Karim Noui for discussions. We also thank the various remarks and suggestions that we got from the three anonymous referees. This work was supported in part by the Agence Nationale de la Recherche; grant ANR-06-BLAN-0050. A.P. was supported by l'Institut Universitaire de France. D.P. was supported by Marie Curie EU-NCG network.

\section{References and Notes}

1. Reid, M.J. Is there a supermassive black hole at the center of the milky way? Int. J. Mod. Phys. D 2009, 18, 889-910.

2. Mueller, A. Experimental evidence of black holes. PoS 2006, P2GC, 017.

3. Broderick, A.E.; Loeb, A.; Narayan, R. The event horizon of Sagittarius A*. Astrophys. J. 2009, 701, 1357-1366.

4. Bekenstein, J. D. Black holes and entropy. Phys. Rev. D 1973, 7, 2333-2346.

5. Hawking, S.W. Particle creation by black holes. Commun. Math. Phys. 1975, 43, 199-220.

6. Thiemann, T. Modern Canonical Quantum GR; Cambridge University Press: Cambridge, UK, 2007.

7. Rovelli, C. Quantum Gravity; Cambridge University Press: Cambridge, UK, 2004.

8. Ashtekar, A.; Lewandowski, J. Background independent quantum gravity: A status report. Class. Quant. Grav. 2004, 21, R53.

9. Perez, A. Introduction to loop quantum gravity and spin foams. In Proceedings of the International Conference on Fundamental Interactions, Domingos Martins, Brazil, 6-12 June 2004.

10. Ashtekar, A.; Taveras, V.; Varadarajan, M. Information is not lost in the evaporation of 2-dimensional black holes. Phys. Rev. Lett. 2008, 100, 211302.

11. Ashtekar, A.; Corichi, A.; Krasnov, K. Isolated horizons: The classical phase space. Adv. Theor. Math. Phys. 1999, 3, 419-478.

12. Ashtekar, A.; Baez, J.; Krasnov, K. Quantum geometry of isolated horizons and black hole entropy. Adv. Theor. Math. Phys. 2000, 4, 1-94.

13. Meissner, K.A. Black hole entropy in loop quantum gravity. Class. Quant. Grav. 2004, 21, 5245-5252.

14. Domagala, M.; Lewandowski, J. Black hole entropy from quantum geometry. Class. Quant. Grav. 2004, 21, 5233-5244.

15. Ghosh, A.; Mitra, P. A bound on the log correction to the black hole area law. Phys. Rev. D 2005, $71,027502$.

16. Gour, G. Algebraic approach to quantum black holes: Logarithmic corrections to black hole entropy. Phys. Rev. D 2002, 66, 104022.

17. Agullo, I.; Barbero, J.F.; Diaz-Polo, J.; Fernandez-Borja, E.; Villasenor, E.J.S. Black hole state counting in LQG: A number theoretical approach. Phys. Rev. Lett. 2008, 100, 211301.

18. Barbero, J.F.; Villasenor, E.J.S. Generating functions for black hole entropy in loop quantum gravity. Phys. Rev. D $2008,77,121502$. 
19. Barbero, J.F.; Villasenor, E.J.S. On the computation of black hole entropy in loop quantum gravity. Class. Quant. Grav. 2009, 26, 035017.

20. Agullo, I.; Barbero, J.F.; Borja, E.F.; Diaz-Polo, J.; Villasenor, E.J.S. The combinatorics of the SU(2) black hole entropy in loop quantum gravity. Phys. Rev. D 2009, 80, 084006.

21. Agullo, I.; Barbero, J.F.; Borja, E.F.; Diaz-Polo, J.; Villasenor, E.J.S. Detailed black hole state counting in loop quantum gravity. Phys. Rev. D 2010, 82, 084029.

22. Engle, J.; Perez, A.; Noui, K. Black hole entropy and SU(2) Chern-Simons theory. Phys. Rev. Lett. 2010, 105, 031302.

23. Engle, J.; Perez, A.; Noui, K.; Pranzetti, D. Black hole entropy from an SU(2)-invariant formulation of type I isolated horizons. Phys. Rev. D 2010, 82, 044050.

24. Basu, R.; Kaul, R.K.; Majumdar, P. Entropy of isolated horizons revisited Phys. Rev. D 2010, 82, 024007.

25. Kaul, R.K.; Majumdar, P. Quantum black hole entropy. Phys. Lett. B 1998, 439, 267.

26. Kaul, R.K.; Majumdar, P. Logarithmic correction to the Bekenstein-Hawking entropy. Phys. Rev. Lett. 2000, 84, 5255.

27. Carlip, S. Black hole thermodynamics and statistical mechanics. Lect. Notes Phys. 2009, 769, 89.

28. Carlip, S. Entropy from conformal field theory at Killing horizons. Class. Quant. Grav. 1999, 16, 3327.

29. Carlip, S. Black hole entropy from conformal field theory in any dimension. Phys. Rev. Lett. 1999, $82,2828$.

30. Carlip, S. Logarithmic corrections to black hole entropy from the Cardy formula. Class. Quant. Grav. 2000, 17, 4175.

31. Livine, E.R.; Terno, D.R. Quantum black holes: Entropy and entanglement on the horizon. Nucl. Phys. B 2006, 741, 131.

32. Ashtekar, A.; Beetle, C.; Lewandowski, J. Geometry of generic isolated horizons. Class. Quant. Grav. 2002, 19, 1195.

33. Ashtekar, A.; Beetle, C.; Dreyer, O.; Fairhurst, S.; Krishnan, B.; Lewandowski, J.; Wisniewski, J. Generic isolated horizons and their applications. Phys. Rev. Lett. 2000, 85, 3564-3567.

34. Ashtekar, A.; Engle, J.; Van Den Broeck, C. Quantum horizons and black hole entropy: Inclusion of distortion and rotation. Class. Quant. Grav. 2005, 22, L27.

35. Beetle, C.; Engle, J. Generic isolated horizons in loop quantum gravity. Class. Quant. Grav. 2010, 27, 235024.

36. Ashtekar, A.; Fairhurst, S.; Krishnan, B. Isolated horizons: Hamiltonian evolution and the first law. Phys. Rev. D 2000, 62, 104025.

37. Ashtekar, A.; Beetle, C; Lewandowski, J. Mechanics of rotating isolated horizons. Phys. Rev. D 2001, 64, 044016.

38. Ashtekar, A.; Engle, J.; Van Den Broeck, C. Quantum horizons and black hole entropy: Inclusion of distortion and rotation. Class. Quant. Grav. 2005, 22, L27.

39. Geroch, R.P.; Held, A.; Penrose, R. A space-time calculus based on pairs of null directions. J. Math. Phys. 1973, 14, 874.

40. Chandrasekhar, S. The Mathematical Theory of Black Holes; Clarendon: Oxford, UK, 1992. 
41. Crnkovic, C.; Witten, E. Covariant description of canonical formalism in geometrical theories. In Three Hundred Years of Gravitation, Print-86-1309 (PRINCETON); Hawking, S.W., Israel, W., Eds.; Cambridge University Press: Cambridge, UK, 1989.

42. Lee, J.; Wald, R.M. Local symmetries and constraints. J. Math. Phys. 1990, 31, 725.

43. Corichi, A.; Wilson-Ewing, E. Surface terms, asymptotics and thermodynamics of the holst action. Class. Quant. Grav. 2010, 27, 205015.

44. Lewandowski, J.; Okolow, A.; Sahlmann, H.; Thiemann, T. Uniqueness of the diffeomorphism invariant state on the quantum holonomy-flux algebra. Commun. Math. Phys. 2006, 267, 703-733.

45. Fleischhack, C. Representations of the Weyl algebra in quantum geometry. Commun. Math. Phys. 2009, 285, 67.

46. Freidel, L.; Perez, A. PI, Waterloo, Canada; CPT, Marseille, France. Unpublished work, 2011.

47. Witten, E. Quantum field theory and the Jones polynomial. Commun. Math. Phys. 1989, 121, 351.

48. Ashtekar, A. Lectures on Nonperturbative Canonical Gravity; World Scientific: Singapore, 1991.

49. Ghosh, A.; Mitra, P. Counting black hole microscopic states in loop quantum gravity. Phys. Rev. D 2006, 74, 064026.

50. Ooguri, H.; Sasakura, N. Discrete and continuum approaches to three-dimensional quantum gravity. Mod. Phys. Lett. A 1991, 6, 3591.

51. Archer, F.; Williams, R.M. The Turaev-Viro state sum model and three-dimensional quantum gravity. Phys. Lett. B 1991, 273, 438.

52. Thiemann, T. Quantum spin dynamics. VIII: The master constraint. Class. Quant. Grav. 2006, 23, 2249.

53. Thiemann, T. The Phoenix project: Master constraint programme for loop quantum gravity. Class. Quant. Grav. 2006, 23, 2211.

54. Major, S.A. Shape in an Atom of Space: Exploring quantum geometry phenomenology. Class. Quant. Grav. 2010, 27, 225012.

55. Engle, J.; Livine, E.; Pereira, R.; Rovelli, C. LQG vertex with finite Immirzi parameter. Nucl. Phys. B 2008, 799, 136.

56. Freidel, L.; Krasnov, K. A new spin foam model for 4d gravity. Class. Quant. Grav. 2008, 25, 125018.

57. Ding, Y.; Han, M.; Rovelli, C. Generalized spinfoams. arXiv 2010, arXiv:gr-qc/10112149.

58. Rezende, D.J.; Perez, A. 4d Lorentzian Holst action with topological terms. Phys. Rev. D 2009, 79, 064026.

59. Engle, J.; Perez, A.; Noui, K.; Pranzetti, D. The $S U(2)$ black hole entropy revisited. arXiv 2011, arXiv:gr-qc/1103.2723.

60. Notice, that the family of descriptions here presented are labelled by an integer $k \in \mathbb{N}$ and a correlated real number. This seem to be the structure that one would wish in order to establish a link between LQG and some interesting speculative ideas explored recently [62].

61. It interesting to speculate a possible understanding of our result from the point of view of renormalization, as emphasized by Jacobson in [63].

62. Sahlmann, H. Newton's constant from a minimal length: Additional models. Class. Quant. Grav. 2011, 28, 015006. 
63. Jacobson, T. Renormalization and black hole entropy in loop quantum gravity. Class. Quant. Grav. 2007, 24, 4875.

(c) 2011 by the authors; licensee MDPI, Basel, Switzerland. This article is an open access article distributed under the terms and conditions of the Creative Commons Attribution license (http://creativecommons.org/licenses/by/3.0/.) 